\begin{tabular}{|c|l|}
\hline Title & Transition of global dynamics of a polygonal vortex ring on a sphere with pole vortices \\
\hline Author(s) & SAKA JO, Takashi \\
\hline Citation & $\begin{array}{l}\text { Physica D: Nonlinear Phenomena, 196/3-4), 243-264 } \\
\text { https://doi.org/L0.1016/.physd.2004.05.009 }\end{array}$ \\
\hline Issue Date & 2004_09 \\
\hline Doc URL & http://hdl.handle.net/2115/8558 \\
\hline Type & article(author version) \\
\hline File Information & PhysicaD_Sakajo_1.pdf \\
\hline
\end{tabular}

Instructions for use 


\title{
Transition of global dynamics of a polygonal vortex ring on a sphere with pole vortices
}

\author{
Takashi SAKAJO \\ Department of Mathematics, Hokkaido University, \\ Sapporo Hokkaido 060-8610, JAPAN. \\ Tel: +81-11-706-4660, Fax: +81-11-727-3705, \\ E-mail:sakajo@math.sci.hokudai.ac.jp.
}

\begin{abstract}
We study the motion of a polygonal ring consists of identical vortex points that are equally spaced at a line of latitude on a sphere with vortex points fixed at the both poles. First, we calculate explicitly all the eigenvalues and the eigenvectors corresponding to them for the linearized problem, from which we consider the stability of the polygonal vortex ring in the presence of the pole vortices. Next, when the number of the vortex points is even in particular, the equations of the vortex points are reduced to those for a pair of two vortex points by assuming a special symmetry. Studying the reduced system mathematically and numerically, we describe an universal transition of global periodic motion of the perturbed polygonal ring. Moreover, we also discuss the stability of the periodic motion.
\end{abstract}

\section{Introduction}

We consider the motion of incompressible and inviscid fluids on a sphere. It helps us understand basic dynamical process observed in many atmospheric phenomena on Earth. In particular, local regions where the vorticity is extremely dense are of importance, since they dominate global structures of the flows. Simple examples of such singular vorticity regions are a vortex point in which the vorticity concentrates discretely and a vortex sheet which is defined as a surface of discontinuity of the velocity field.

The author has investigated evolution of a vortex sheet on the sphere with vortex points that are fixed at the both poles[16]. Mathematical analysis and numerical computation indicate that the linear stability of the vortex sheet changes due to the pole vortices and consequently it evolves into a structure composed of many rollingup spirals. Figure 1 shows some snapshots of the vortex sheet after a long time evolution for various strengths of the pole vortices, when the stationary vortex sheet at the line of latitude $\theta_{0}=\frac{\pi}{3}$ is slightly perturbed at an initial moment. The centers of the rolling-up spirals are arranged along a line of latitude, while the size and the number of the spirals are different depending on the strengths of the pole vortices. An original motivation of the present study is to understand long time evolution of the structure of the rolling-up spirals. However, since the outer turns of the spirals interact strongly as soon as they approach each other, it is too complicated to deal with the evolution as it stands. Therefore, as a simplified mathematical model, assuming that the circulation contained in the spiral concentrates in the center point, we consider the evolution of $N$-vortex points that are equally spaced at the line of latitude on the sphere, which we refer to as a $N$-ring. In the present 
paper, we focus especially on global dynamics of the $N$-ring when it is perturbed slightly.

Now, let $\left(\Theta_{m}, \Psi_{m}\right)$ denote the position of the $m$ th vortex point in the spherical coordinates. The strengths of the north and the south pole vortices are expressed by $\Gamma_{1}$ and $\Gamma_{2}$, respectively. Furthermore, we assume that the strengths of the vortex points are identical with each other, that is $\Gamma=\frac{2 \pi}{N}$. Then, the motion of the $N$ vortex points is Hamiltonian dynamical system[8, 12], whose Hamiltonian is given by

$$
\begin{aligned}
H= & -\frac{\Gamma^{2}}{8 \pi} \sum_{m=1}^{N} \sum_{j \neq m}^{N} \log \left(1-\cos \gamma_{m j}\right) \\
& -\frac{\Gamma_{1} \Gamma}{4 \pi} \sum_{m=1}^{N} \log \left(1-\cos \Theta_{m}\right)-\frac{\Gamma_{2} \Gamma}{4 \pi} \sum_{m=1}^{N} \log \left(1+\cos \Theta_{m}\right),
\end{aligned}
$$

in which $\gamma_{m j}$ represents the central angle between the $m$ th and the $j$ th vortex points, and

$$
\cos \gamma_{m j}=\cos \Theta_{m} \cos \Theta_{j}+\sin \Theta_{m} \sin \Theta_{j} \cos \left(\Psi_{m}-\Psi_{j}\right) .
$$

With certain canonical variables[12], the equations of the $N$-vortex points on the sphere with the pole vortices are derived from the Hamiltonian as follows:

$$
\begin{aligned}
\dot{\Theta}_{m}= & -\frac{\Gamma}{4 \pi} \sum_{j \neq m}^{N} \frac{\sin \Theta_{j} \sin \left(\Psi_{m}-\Psi_{j}\right)}{1-\cos \gamma_{m j}}, \\
\sin \Theta_{m} \dot{\Psi}_{m}= & -\frac{\Gamma}{4 \pi} \sum_{j \neq m}^{N} \frac{\cos \Theta_{m} \sin \Theta_{j} \cos \left(\Psi_{m}-\Psi_{j}\right)-\sin \Theta_{m} \cos \Theta_{j}}{1-\cos \gamma_{m j}} \\
& +\frac{\Gamma_{1}}{4 \pi} \frac{\sin \Theta_{m}}{1-\cos \Theta_{m}}-\frac{\Gamma_{2}}{4 \pi} \frac{\sin \Theta_{m}}{1+\cos \Theta_{m}}, \quad m=1,2, \cdots, N .
\end{aligned}
$$

Let us briefly survey some of the recent research in terms of the $N$-vortex points on the sphere. As for a complete review of general $N$-vortex problems, refer to the book by Newton[12]. The motion of three vortex points on the sphere without pole vortices is integrable and its geometric structure of streamline is wellstudied $[4,5,6,15]$. Pekarsky and Marsden[13] studied a nonlinear stability for the 3 -ring of vortex points with arbitrary strengths. While the motion of the $N$-vortex points for $N>3$ is nonintegrable in general, there are some papers studying the relative equilibria by using the $S O(3)$-symmetry of the Hamiltonian[7, 10]. Lim, Montaldi and Roberts[11] gave a clear classification of the relative equilibria of arbitrary number of identical vortex points, in which the $N$-ring is included. LaurentPoltz considered a system composed of $2 N$-vortex points whose strengths alternate between +1 and -1 . He gave some relative fixed configurations and examined their stability by using the energy momentum method. As for the $N$-ring of identical vortex points, linear and nonlinear stability analysis have been done by Boatto and Cabral[1] in connection with the stability of the polygonal vortex ring on the plane[2] and of vortex patches on the sphere[3]. Specifically, they derived a sufficient condition that the Hessian of Hamiltonian evaluated at the $N$-ring is positive or negative definite, and consequently they gave the complete stability analysis for the polygonal configuration for $3 \leq N \leq 7$.

The paper consists of five sections. In $\S 2$, we carry out the linear stability analysis for the $N$-ring again, although the stability condition has already been given in the preceding paper[1]. This is because we would like to give not only the stability condition of the $N$-ring on the sphere in the presence of the pole vortices, 

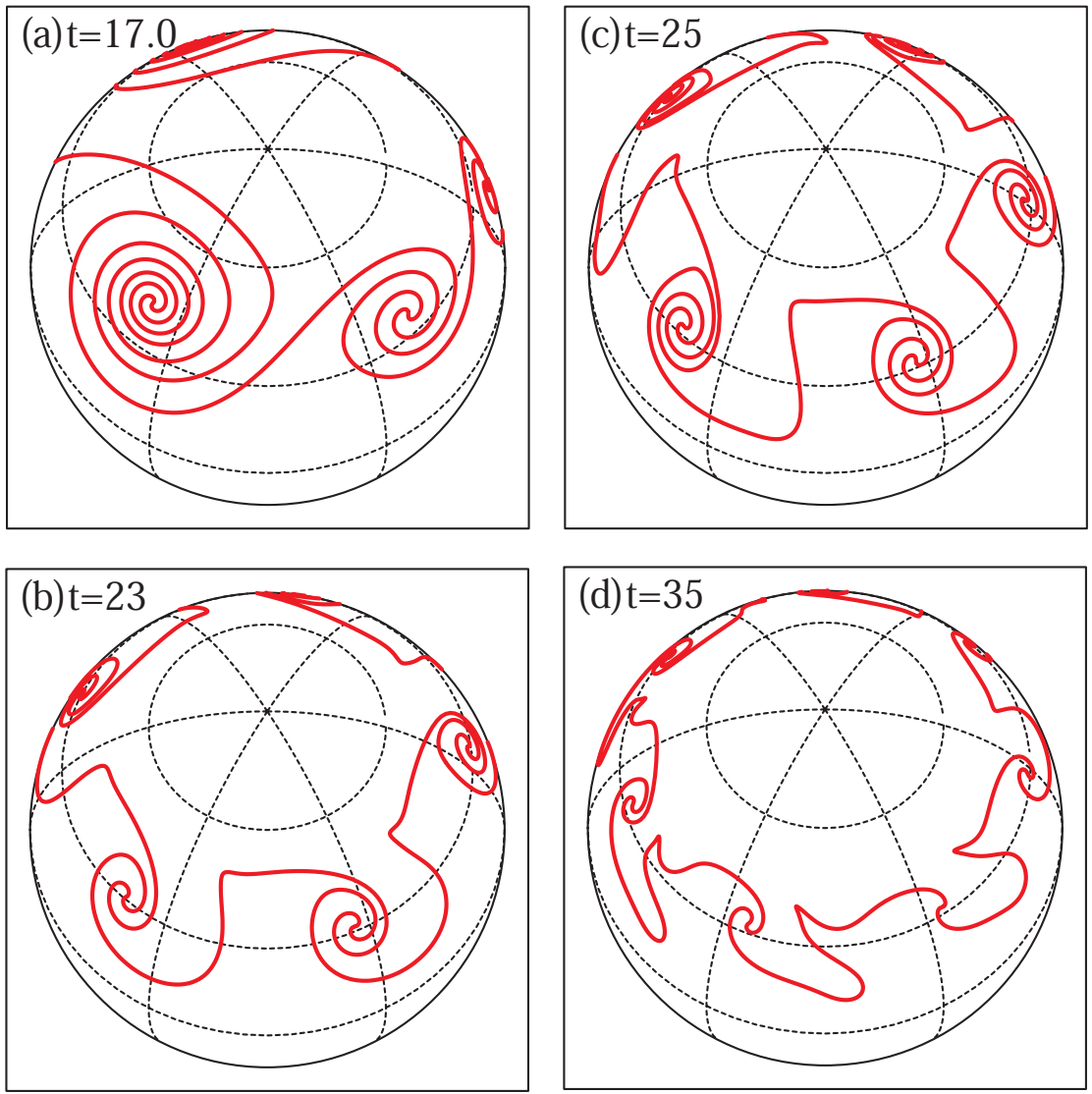

Figure 1: Structures of the vortex sheet after a long time evolution for the various strengths of the north pole vortex $\Gamma_{1}$ and the south pole vortex $\Gamma_{2}$. The vortex sheet is initially located at the line of latitude $\theta_{0}=\frac{\pi}{3}$ on the sphere. (a) Four spirals at $t=17$ for $\Gamma_{1}=0$ and $\Gamma_{2}=0$, (b) Five spirals at $t=23$ for $\Gamma_{1}=1.5 \pi$ and $\Gamma_{2}=-3.5 \pi$, (c) Five spirals at $t=25$ for $\Gamma_{1}=\pi$ and $\Gamma_{2}=\pi$, and (d) Seven spirals at $t=35$ for $\Gamma_{1}=2.5 \pi$ and $\Gamma_{2}=-4.5 \pi$. 
but also all the eigenvalues and the eigenvectors corresponding to them explicitly, which play an indispensable role to describe long time evolution of the perturbed $N$-ring in the following sections. In $\S 3$, when the number of vortex points is even, we reduce the equations of the $N$-vortex points (2) and (3) to those for a pair of two vortex points on the assumption that the $N$-ring has an alternately pairing symmetry. The phase space of the reduced system is equivalent to the eigenspace corresponding to one of the eigenvalues. Mathematical analysis of the 4-ring at the equator is accomplished in $\S 4$. We classify dynamics of the reduced system into five types and then describe a transition of global periodic behavior of the perturbed 4-ring and its stability. In addition, we show numerically that the same transition of global dynamics is observed universally regardless of the number of vortex points and the position of the $N$-ring. Finally, we organize the results concisely in the last section.

\section{Eigenvalue problem for the $N$-ring}

First of all, we review some known facts in terms of the cyclic matrix that is defined as follows:

Definition 1 A matrix $X$ is called cyclic, if it is represented by

$$
X=\left(\begin{array}{ccccc}
x_{0} & x_{1} & x_{2} & \cdots & x_{N-1} \\
x_{N-1} & x_{0} & x_{1} & \cdots & x_{N-2} \\
x_{N-2} & x_{N-1} & x_{0} & \cdots & x_{N-3} \\
\vdots & \vdots & \vdots & \ddots & \vdots \\
x_{1} & x_{2} & x_{3} & \cdots & x_{0}
\end{array}\right)
$$

It is easy to see the following property holds.

Lemma 2 If $X$ and $Y$ are cyclic, then $c X(c \in \mathbf{R}), X+Y$ and $X Y$ are also cyclic.

It is also shown that the determinant of the cyclic matrix is expressed by the components in the first row.

Lemma 3 If $X$ is cyclic, then its determinant is given by

$$
\operatorname{det}(X)=\prod_{p=0}^{N-1}\left(x_{0}+\omega_{p} x_{1}+\omega_{p}^{2} x_{2}+\cdots+\omega_{p}^{N-1} x_{N-1}\right), \quad \omega_{p}=e^{\frac{2 \pi i p}{N}},
$$

where $\mathrm{i}$ is the complex unit.

Second, we give the linearized equations for the $N$-ring at the line of latitude $\theta_{0}$, which is represented by

$$
\Theta_{m}=\theta_{0}, \quad \Psi_{m}=\frac{2 \pi m}{N}, \quad m=1,2, \cdots, N .
$$

It follows from the (2), (3), and (5) that we have

$$
\dot{\Theta}_{m}=0, \quad \dot{\Psi}_{m}=V_{0}(N),
$$

where

$$
V_{0}(N)=\frac{\Gamma_{1}-\Gamma_{2}}{4 \pi \sin ^{2} \theta_{0}}+\frac{\left(\Gamma_{1}+\Gamma_{2}+2 \pi\right) \cos \theta_{0}}{4 \pi \sin ^{2} \theta_{0}}-\frac{1}{2 N} \frac{\cos \theta_{0}}{\sin ^{2} \theta_{0}} .
$$


Hence, the $N$-ring is a steady solution in the spherical coordinates rotating with the constant speed $V_{0}(N)$ in the longitudinal direction. Now, we add a small disturbance to the steady state, namely for $\epsilon \ll 1$,

$$
\Theta_{m}(t)=\theta_{0}+\epsilon \theta_{m}(t), \quad \Psi_{m}(t)=\frac{2 \pi m}{N}+V_{0}(N) t+\epsilon \varphi_{m}(t) .
$$

Then, substituting (6) into the equations (2) and (3), we obtain the linearized equations of $O(\epsilon)$ as follows:

$$
\begin{aligned}
\dot{\theta}_{m} & =\frac{1}{2 N \sin \theta_{0}} \sum_{j \neq m}^{N} \frac{\varphi_{m}-\varphi_{j}}{1-\cos \frac{2 \pi}{N}(m-j)}, \\
\dot{\varphi}_{m} & =\frac{1}{2 N \sin ^{3} \theta_{0}} \sum_{j \neq m}^{N} \frac{\theta_{m}-\theta_{j}}{1-\cos \frac{2 \pi}{N}(m-j)}+B_{N} \theta_{m},
\end{aligned}
$$

in which

$$
B_{N}=\frac{1+\cos ^{2} \theta_{0}}{2 N \sin ^{3} \theta_{0}}-\frac{\left(\Gamma_{1}-\Gamma_{2}\right) \cos \theta_{0}}{2 \pi \sin ^{3} \theta_{0}}-\frac{\left(\Gamma_{1}+\Gamma_{2}+2 \pi\right)\left(1+\cos ^{2} \theta_{0}\right)}{4 \pi \sin ^{3} \theta_{0}} .
$$

Let $\vec{\phi}$ denote the $2 N$-vector ${ }^{t}\left(\theta_{1}, \theta_{2}, \cdots, \theta_{N}, \varphi_{1}, \varphi_{2}, \cdots, \varphi_{N}\right)$. Then, the linearized equations (7) and (8) are rewritten in the following matrix form:

$$
\frac{d \vec{\phi}}{d t}=\left(\begin{array}{cc}
O & A \\
B & O
\end{array}\right) \vec{\phi} \equiv L \vec{\phi}, \quad A=\alpha K, \quad B=\beta K+B_{N} E,
$$

where

$$
\alpha=\frac{1}{2 N \sin \theta_{0}}, \quad \beta=\frac{1}{2 N \sin ^{3} \theta_{0}},
$$

and the symmetric matrix $K$ is represented by

$$
K=\left(\begin{array}{cccc}
\sum_{j \neq 1}^{N} \frac{1}{1-\cos \frac{2 \pi}{N}(1-j)} & \frac{-1}{1-\cos \frac{2 \pi}{N}} & \cdots & \frac{-1}{1-\cos \frac{2 \pi}{N}(1-N)} \\
\frac{-1}{1-\cos \frac{2 \pi}{N}} & \sum_{j \neq 2}^{N} \frac{1-\cos \frac{2 \pi}{N}(2-j)}{1-\cos \frac{2 \pi}{N}(2-N)} & \cdots & \vdots \\
\vdots & \vdots & \ddots & \vdots \\
\frac{-1}{1-\cos \frac{2 \pi}{N}(N-1)} & \frac{-1}{1-\cos \frac{2 \pi}{N}(N-2)} & \cdots & \sum_{j \neq N}^{N} \frac{1}{1-\cos \frac{2 \pi}{N}(N-j)}
\end{array}\right) .
$$

As a matter of fact, the matrix $K$ is cyclic. Indeed, when we define $k_{m}$ by

$$
k_{0}=\sum_{j=1}^{N-1} \frac{1}{1-\cos \frac{2 \pi j}{N}}, \quad k_{m}=\frac{-1}{1-\cos \frac{2 \pi m}{N}}, \quad m=1,2, \cdots, N-1,
$$

the first row of $K$ is expressed by $\left(k_{0}, k_{1}, \cdots, k_{N-1}\right)$ and besides $k_{m}$ has the following symmetry

$$
k_{m}=k_{N-m}, \quad m=1,2, \cdots, N-1,
$$

which indicates that the matrix $K$ is cyclic.

Third, we calculate the eigenvalue $\lambda$ of the linearized matrix $L$. The characteristic equation becomes

$$
\operatorname{det}\left(L-\lambda E_{2 N}\right)=\operatorname{det}\left(\begin{array}{cc}
-\lambda E_{N} & A \\
B & -\lambda E_{N}
\end{array}\right)=\operatorname{det}\left(\lambda^{2} E_{N}-A B\right)=0,
$$

in which $E_{N}$ is the $N \times N$ unit matrix. Since $K$ is cyclic and $P \equiv \lambda^{2} E_{N}-A B$ is represented by $\lambda^{2} E_{N}-\alpha K\left(\beta K+B_{N} E_{N}\right), P$ is also cyclic due to Lemma 2. Hence, it follows from Lemma 3 that the characteristic equation of $P$ is equivalent to

$$
p_{0}+\omega_{p} p_{1}+\omega_{p}^{2} p_{2}+\cdots+\omega_{p}^{N-1} p_{N-1}=0, \quad p=0,1, \cdots, N-1,
$$


in which $p_{m}$ represents the $m$ th component of the first row of the matrix $P$.

Since both $A=\alpha K$ and $B=\beta K+B_{N} E_{N}$ are cyclic, the first row of the matrix $A$ and the $m$ th column of the matrix $B$ are represented by

$$
\begin{aligned}
\vec{a}_{1} & =\left(\alpha k_{0}, \alpha k_{1}, \cdots, \alpha k_{N-1}\right), \\
\vec{b}_{m} & ={ }^{t}\left(\beta k_{m-1}, \beta k_{m-2}, \cdots, \beta k_{1}, \beta k_{0}+B_{N}, \beta k_{N-1}, \cdots, \beta k_{m}\right),
\end{aligned}
$$

respectively. Thus, $P=\lambda^{2} E_{N}-A B$ leads to $p_{m}=\lambda^{2} \delta_{m 0}-\vec{a}_{1} \vec{b}_{m+1}$, in which $\delta_{m 0}$ is Kronecker's delta. Hence, noting that $\omega_{p}^{-m}=\omega_{p}^{N-m}$, we solve the characteristic equations (11) for $p=0,1, \cdots, N-1$ :

$$
\begin{aligned}
\lambda^{2} & =\vec{a}_{1} \vec{b}_{1}+\omega_{p} \vec{a}_{1} \vec{b}_{2}+\cdots+\omega_{p}^{N-1} \vec{a}_{1} \vec{b}_{N} \\
& =\vec{a}_{1}\left(\vec{b}_{1}+\omega_{p} \vec{b}_{2}+\cdots+\omega_{p}^{N-1} \vec{b}_{N}\right) \\
& =\left(\beta k_{0}+B_{N}+\omega_{p} \beta k_{1}+\cdots+\omega_{p}^{N-1} \beta k_{N-1}\right) \vec{a}_{1}{ }^{t}\left(1, \omega_{p}, \cdots, \omega_{p}^{N-1}\right) \\
& =\left(\beta k_{0}+B_{N}+\omega_{p} \beta k_{1}+\cdots+\omega_{p}^{N-1} \beta k_{N-1}\right)\left(\alpha k_{0}+\omega_{p} \alpha k_{1}+\cdots+\omega_{p}^{N-1} \alpha k_{N-1}\right) \\
& =\xi_{p} \eta_{p},
\end{aligned}
$$

where $\xi_{p}$ and $\eta_{p}$ are defined by

$$
\begin{aligned}
\xi_{p} & =\alpha\left(k_{0}+\omega_{p} k_{1}+\cdots+\omega_{p}^{N-1} k_{N-1}\right), \\
\eta_{p} & =\beta\left(k_{0}+\omega_{p} k_{1}+\cdots+\omega_{p}^{N-1} k_{N-1}\right)+B_{N} .
\end{aligned}
$$

In addition, since we have

$$
k_{0}+\omega_{p} k_{1}+\cdots+\omega_{p}^{N-1} k_{N-1}=\sum_{j=1}^{N-1} \frac{1-\omega_{p}^{j}}{1-\cos \frac{2 \pi j}{N}}=p(N-p),
$$

the eigenvalues are given explicitly as follows.

Theorem 4 For $p=0,1, \cdots, N-1$, the eigenvalues $\lambda_{p}^{ \pm}$of the matrix $L$ are represented by

$$
\lambda_{p}^{ \pm}= \pm \sqrt{\alpha \beta\left(p N-p^{2}\right)^{2}+\alpha B_{N}\left(p N-p^{2}\right)} .
$$

Now, let $M$ be defined by $N=2 M$ for even $N$ and by $N=2 M+1$ for odd $N$. Then, Theorem 4 directly reveals that the eigenvalues satisfy the following symmetry.

Corollary $5 \lambda_{0}^{ \pm}=0$ and $\lambda_{p}^{ \pm}=\lambda_{N-p}^{ \pm}$for $p=1,2, \cdots, M$.

Therefore, when $N$ is even, the eigenvalues $\lambda_{M}^{ \pm}$are simple, while the others are double. On the other hand, all the eigenvalues are double for odd $N$.

It also follows from Theorem 4 that the stability condition for the eigenvalues are obtained.

Theorem 6 For $p=1,2, \cdots, M$, the eigenvalues $\lambda_{p}^{ \pm}$are neutrally stable if

$$
\frac{p N-p^{2}}{N}+\frac{1+\cos ^{2} \theta_{0}}{N}<\kappa_{1}\left(1+\cos ^{2} \theta_{0}\right)+\kappa_{2} \cos \theta_{0},
$$

where the parameters $\kappa_{1}$ and $\kappa_{2}$ are defined by the strengths of the pole vortices as follows:

$$
\kappa_{1}=\frac{\Gamma_{1}+\Gamma_{2}+2 \pi}{2 \pi}, \quad \kappa_{2}=\frac{\Gamma_{1}-\Gamma_{2}}{\pi} .
$$


Proof: It follows from Theorem 4 and (9) that we have

The eigenvalues $\lambda_{p}^{ \pm}$are neutrally stable

$$
\begin{aligned}
& \Longleftrightarrow\left(\lambda_{p}^{ \pm}\right)^{2}=\alpha \beta\left(p N-p^{2}\right)^{2}+\alpha B_{N}\left(p N-p^{2}\right)<0 \\
& \Longleftrightarrow \beta\left(p N-p^{2}\right)+B_{N}<0 \\
& \Longleftrightarrow \frac{p N-p^{2}}{2 N \sin ^{3} \theta_{0}}+\frac{1+\cos ^{2} \theta_{0}}{2 N \sin ^{3} \theta_{0}}-\frac{\left(\Gamma_{1}-\Gamma_{2}\right) \cos \theta_{0}}{2 \pi \sin ^{3} \theta_{0}}-\frac{\left(\Gamma_{1}+\Gamma_{2}+2 \pi\right)\left(1+\cos ^{2} \theta_{0}\right)}{4 \pi \sin ^{3} \theta_{0}}<0 \\
& \Longleftrightarrow(15) . \quad \square
\end{aligned}
$$

Since the left-hand side of (15) is monotone increasing for $1 \leq p \leq M$, if the eigenvalues $\lambda_{M}^{ \pm}$are neutrally stable, then all the eigenvalues become neutrally stable automatically. Accordingly, the stability condition (15) for $\lambda_{M}^{ \pm}$is equivalent to that for the $N$-ring in the presence of the pole vortices.

Corollary 7 The $N$-ring at the line of latitude $\theta_{0}$ on the sphere with pole vortices is stable if and only if

$$
\begin{aligned}
\frac{N}{4}+\frac{1+\cos ^{2} \theta_{0}}{N} & <\kappa_{1}\left(1+\cos ^{2} \theta_{0}\right)+\kappa_{2} \cos \theta_{0} \text { for } \quad N \text { : even }, \\
\frac{N^{2}-1}{4 N}+\frac{1+\cos ^{2} \theta_{0}}{N} & <\kappa_{1}\left(1+\cos ^{2} \theta_{0}\right)+\kappa_{2} \cos \theta_{0} \text { for } N: \text { odd } .
\end{aligned}
$$

When there is no pole vortex, i.e. $\kappa_{1}=1$ and $\kappa_{2}=0$, then the condition (16) agrees with the stability condition given by Boatto et.al[1] and Polvani et al.[14].

Finally, we obtain all the eigenvectors corresponding to the eigenvalues $\lambda_{p}^{ \pm}$for $p=1,2, \cdots, M$.

Theorem 8 There are two linearly independent eigenvectors $\vec{\phi}_{p}^{ \pm}$and $\vec{\psi}_{p}^{ \pm}$corresponding to the eigenvalues $\lambda_{p}^{ \pm}$, which are given by

$$
\begin{aligned}
\vec{\phi}_{p}^{ \pm} & ={ }^{t}\left(\sqrt{\xi_{p}}, \sqrt{\xi_{p}} \omega_{p}, \cdots, \sqrt{\xi_{p}} \omega_{p}^{N-1}, \pm \sqrt{\eta_{p}}, \pm \sqrt{\eta_{p}} \omega_{p}, \cdots, \pm \sqrt{\eta_{p}} \omega_{p}^{N-1}\right), \\
\vec{\psi}_{p}^{ \pm} & ={ }^{t}\left(\sqrt{\xi_{p}}, \sqrt{\xi_{p}} \bar{\omega}_{p}, \cdots, \sqrt{\xi_{p}} \bar{\omega}_{p}^{N-1}, \pm \sqrt{\eta_{p}}, \pm \sqrt{\eta_{p}} \bar{\omega}_{p}, \cdots, \pm \sqrt{\eta_{p}} \bar{\omega}_{p}^{N-1}\right),
\end{aligned}
$$

where $\bar{\omega}_{p}$ denotes the complex conjugate of $\omega_{p}$.

Proof: Since the eigenvalues $\lambda_{p}^{ \pm}$are expressed by $\pm \sqrt{\xi_{p} \eta_{p}}$ from (12), the $m$ th component of the vector $L \vec{\phi}_{p}^{ \pm}$for $m=1,2, \cdots, N$ becomes

$$
\begin{aligned}
\left(L \vec{\phi}_{p}^{ \pm}\right)_{m} & = \pm \sqrt{\eta_{p}}\left(A^{t}\left(1, \omega_{p}, \cdots, \omega_{p}^{N-1}\right)\right)_{m} \\
& = \pm \sqrt{\eta_{p}} \omega_{p}^{m-1} \alpha\left(k_{0}+\omega_{p} k_{1}+\cdots+\omega_{p}^{N-1} k_{N-1}\right) \\
& = \pm \sqrt{\eta_{p}} \omega_{p}^{m-1} \xi_{p}= \pm \sqrt{\xi_{p} \eta_{p}} \omega_{p}^{m-1} \sqrt{\xi_{p}}=\lambda_{p}^{ \pm}\left(\vec{\phi}_{p}^{ \pm}\right)_{m}
\end{aligned}
$$

For $m=N+1, \cdots, 2 N$, we have

$$
\begin{aligned}
\left(L \vec{\phi}_{p}^{+}\right)_{m} & =\sqrt{\xi_{p}}\left(B^{t}\left(1, \omega_{p}, \cdots, \omega_{p}^{N-1}\right)\right)_{m} \\
& =\sqrt{\xi_{p}} \omega_{p}^{m-1}\left(\beta\left(k_{0}+\omega_{p} k_{1}+\cdots+\omega_{p}^{N-1} k_{N-1}\right)+B_{N}\right) \\
& =\sqrt{\xi_{p}} \omega_{p}^{m-1} \eta_{p}= \pm \sqrt{\xi_{p} \eta_{p}} \pm \omega_{p}^{m-1} \sqrt{\eta_{p}}=\lambda_{p}^{ \pm}\left(\vec{\phi}_{p}^{ \pm}\right)_{m} .
\end{aligned}
$$

On the other hand, since $\xi_{p}$ and $\eta_{p}$ are real due to (13), we note that $\xi_{p}=\alpha\left(k_{0}+\right.$ $\left.\bar{\omega}_{p} k_{1}+\cdots+\bar{\omega}_{p}^{N-1} k_{N-1}\right)$ and $\eta_{p}=\beta\left(k_{0}+\bar{\omega}_{p} k_{1}+\cdots+\bar{\omega}_{p}^{N-1} k_{N-1}\right)+B_{N}$, from which we show similarly that $\vec{\psi}_{p}^{ \pm}$are also the eigenvectors corresponding to $\lambda_{p}^{ \pm}$. 
This theorem indicates that the two eigenvectors of $\lambda_{0}^{ \pm}$are equivalently given by $\vec{\phi}_{0}^{ \pm}=\vec{\psi}_{0}^{ \pm}={ }^{t}\left(0, \cdots, 0, \pm \sqrt{B_{N}}, \cdots \pm \sqrt{B_{N}}\right)$, which represent the rotational vectors in the longitudinal direction. On the other hand, the two eigenvectors corresponding to simple $\lambda_{M}^{ \pm}$for $N=2 M$ are also identically described by

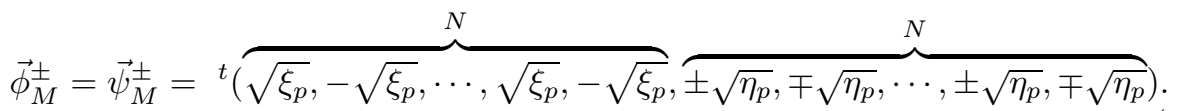

We note that the eigenvectors have an alternately pairing symmetry, i.e. $\dot{\theta}_{2 m-1}=$ $\dot{\theta}_{1}, \dot{\theta}_{2 m}=\dot{\theta}_{2}, \dot{\varphi}_{2 m-1}=\dot{\varphi}_{1}$, and $\dot{\varphi}_{2 m}=\dot{\varphi}_{2}$ for $m=1,2, \cdots, M$.

\section{Reduction of the equations for even vortex points}

When the number of vortex points is even, i.e. $N=2 M$, we reduce the equations (2) and (3) for the $N$-vortex points $\left(\Theta_{m}, \Psi_{m}\right)$ to those for a pair of vortex points $\left(\Theta_{1}, \Psi_{1}\right)$ and $\left(\Theta_{2}, \Psi_{2}\right)$ by assuming the following alternate symmetry:

$$
\begin{aligned}
& \Theta_{2 m-1}=\Theta_{1}, \quad \Psi_{2 m-1}=\Psi_{1}+\frac{2 \pi}{M}(m-1), \\
& \Theta_{2 m}=\Theta_{2}, \quad \Psi_{2 m}=\Psi_{2}+\frac{2 \pi}{M}(m-1), \quad m=1,2, \cdots, M .
\end{aligned}
$$

First, remembering that $\Gamma=\frac{\pi}{M}$, we reduce the Hamiltonian (1) to

$$
\begin{aligned}
H= & -\frac{\pi}{8 M} \sum_{j=2}^{M} \log \left(1-\cos ^{2} \Theta_{1}-\sin ^{2} \Theta_{1} \cos \frac{2 \pi(1-j)}{M}\right) \\
& -\frac{\pi}{8 M} \sum_{j=2}^{M} \log \left(1-\cos ^{2} \Theta_{2}-\sin ^{2} \Theta_{2} \cos \frac{2 \pi(1-j)}{M}\right) \\
& -\frac{\pi}{8 M} \sum_{j=1}^{M} \log \left(1-\cos \Theta_{1} \cos \Theta_{2}-\sin \Theta_{1} \sin \Theta_{2} \cos \left(\Psi_{1}-\Psi_{2}+\frac{2 \pi(1-j)}{M}\right)\right) \\
& -\frac{\pi}{8 M} \sum_{j=1}^{M} \log \left(1-\cos \Theta_{1} \cos \Theta_{2}-\sin \Theta_{1} \sin \Theta_{2} \cos \left(\Psi_{2}-\Psi_{1}+\frac{2 \pi(1-j)}{M}\right)\right) \\
& -\frac{\Gamma_{1}}{4} \log \left(1-\cos \Theta_{1}\right)-\frac{\Gamma_{2}}{4} \log \left(1+\cos \Theta_{1}\right) \\
& -\frac{\Gamma_{1}}{4} \log \left(1-\cos \Theta_{2}\right)-\frac{\Gamma_{2}}{4} \log \left(1+\cos \Theta_{2}\right) .
\end{aligned}
$$

Next, substituting (18) into the equations (2) and (3), we obtain the equations for $\left(\Theta_{1}, \Psi_{1}\right)$ and $\left(\Theta_{2}, \Psi_{2}\right)$.

$$
\begin{aligned}
\dot{\Theta}_{1}= & -\frac{1}{8 M} \sum_{j=1}^{M} \frac{\sin \Theta_{2} \sin \left(\Psi_{1}-\Psi_{2}+\frac{2 \pi(1-j)}{M}\right)}{1-\cos \Theta_{1} \cos \Theta_{2}-\sin \Theta_{1} \sin \Theta_{2} \cos \left(\Psi_{1}-\Psi_{2}+\frac{2 \pi(1-j)}{M}\right)} \\
& +\frac{1}{8 M} \sum_{j=1}^{M} \frac{\sin \Theta_{2} \sin \left(\Psi_{2}-\Psi_{1}+\frac{2 \pi(1-j)}{M}\right)}{1-\cos \Theta_{1} \cos \Theta_{2}-\sin \Theta_{1} \sin \Theta_{2} \cos \left(\Psi_{2}-\Psi_{1}+\frac{2 \pi(1-j)}{M}\right)} \\
\dot{\Theta}_{2}= & -\frac{1}{8 M} \sum_{j=1}^{M} \frac{\sin \Theta_{1} \sin \left(\Psi_{2}-\Psi_{1}+\frac{2 \pi(1-j)}{M}\right)}{1-\cos \Theta_{1} \cos \Theta_{2}-\sin \Theta_{1} \sin \Theta_{2} \cos \left(\Psi_{2}-\Psi_{1}+\frac{2 \pi(1-j)}{M}\right)}
\end{aligned}
$$




$$
\begin{aligned}
& +\frac{1}{8 M} \sum_{j=1}^{M} \frac{\sin \Theta_{1} \sin \left(\Psi_{1}-\Psi_{2}+\frac{2 \pi(1-j)}{M}\right)}{1-\cos \Theta_{1} \cos \Theta_{2}-\sin \Theta_{1} \sin \Theta_{2} \cos \left(\Psi_{1}-\Psi_{2}+\frac{2 \pi(1-j)}{M}\right)}, \quad(21) \\
\dot{\Psi}_{1}= & -\frac{1}{8 M \sin \Theta_{1}} \sum_{j=1}^{M} \frac{\cos \Theta_{1} \sin \Theta_{2} \cos \left(\Psi_{1}-\Psi_{2}+\frac{2 \pi(1-j)}{M}\right)-\sin \Theta_{1} \cos \Theta_{2}}{1-\cos \Theta_{1} \cos \Theta_{2}-\sin \Theta_{1} \sin \Theta_{2} \cos \left(\Psi_{1}-\Psi_{2}+\frac{2 \pi(1-j)}{M}\right)} \\
& -\frac{1}{8 M \sin \Theta_{1}} \sum_{j=1}^{M} \frac{\cos \Theta_{1} \sin \Theta_{2} \cos \left(\Psi_{2}-\Psi_{1}+\frac{2 \pi(1-j)}{M}\right)-\sin \Theta_{1} \cos \Theta_{2}}{1-\cos \Theta_{1} \cos \Theta_{2}-\sin \Theta_{1} \sin \Theta_{2} \cos \left(\Psi_{2}-\Psi_{1}+\frac{2 \pi(1-j)}{M}\right)} \\
& +\left(\frac{1}{4}-\frac{1}{4 M}+\frac{\Gamma_{1}+\Gamma_{2}}{4 \pi}\right) \frac{\cos \Theta_{1}}{\sin \Theta_{1}}+\frac{\Gamma_{1}-\Gamma_{2}}{4 \pi} \frac{1}{\sin \Theta_{1}}, \\
\dot{\Psi}_{2}= & -\frac{1}{8 M \sin \Theta_{2}} \sum_{j=1}^{M} \frac{\cos \Theta_{2} \sin \Theta_{1} \cos \left(\Psi_{2}-\Psi_{1}+\frac{2 \pi(1-j)}{M}\right)-\sin \Theta_{2} \cos \Theta_{1}}{1-\cos \Theta_{1} \cos \Theta_{2}-\sin \Theta_{1} \sin \Theta_{2} \cos \left(\Psi_{2}-\Psi_{1}+\frac{2 \pi(1-j)}{M}\right)} \\
& -\frac{1}{8 M \sin \Theta_{2}} \sum_{j=1}^{M} \frac{\cos \Theta_{2} \sin \Theta_{1} \cos \left(\Psi_{1}-\Psi_{2}+\frac{2 \pi(1-j)}{M}\right)-\sin \Theta_{2} \cos \Theta_{1}}{1-\cos \Theta_{1} \cos \Theta_{2}-\sin \Theta_{1} \sin \Theta_{2} \cos \left(\Psi_{1}-\Psi_{2}+\frac{2 \pi(1-j)}{M}\right)} \\
& +\left(\frac{1}{4}-\frac{1}{4 M}+\frac{\Gamma_{1}+\Gamma_{2}}{4 \pi}\right) \frac{\cos \Theta_{2}}{\sin ^{2} \Theta_{2}}+\frac{\Gamma_{1}-\Gamma_{2}}{4 \pi} \frac{1}{\sin ^{2} \Theta_{2}} .
\end{aligned}
$$

It follows from $(20)$ and $(21)$ that $\cos \Theta_{1}+\cos \Theta_{2}$ is constant. Since we are interested in the motion of the perturbed $N$-ring at the line of latitude $\theta_{0}$, the invariant is given by

$$
\cos \Theta_{1}+\cos \Theta_{2}=2 \cos \theta_{0} .
$$

Furthermore, the equations (22) and (23) are reduced to that for $\Phi=\Psi_{1}-\Psi_{2}$.

$$
\begin{aligned}
\dot{\Phi}= & -\frac{\cos \Theta_{1}-\cos \Theta_{2}}{8 M \sin \Theta_{1} \sin \Theta_{2}} \sum_{j=1}^{M} \frac{\sin \Theta_{1} \sin \Theta_{2}+\left(1+\cos \Theta_{1} \cos \Theta_{2}\right) \cos \left(\Phi+\frac{2 \pi(1-j)}{M}\right)}{1-\cos \Theta_{1} \cos \Theta_{2}-\sin \Theta_{1} \sin \Theta_{2} \cos \left(\Phi+\frac{2 \pi(1-j)}{M}\right)} \\
& -\frac{\cos \Theta_{1}-\cos \Theta_{2}}{8 M \sin \Theta_{1} \sin \Theta_{2}} \sum_{j=1}^{M} \frac{\sin \Theta_{1} \sin \Theta_{2}+\left(1+\cos \Theta_{1} \cos \Theta_{2}\right) \cos \left(-\Phi+\frac{2 \pi(1-j)}{M}\right)}{1-\cos \Theta_{1} \cos \Theta_{2}-\sin \Theta_{1} \sin \Theta_{2} \cos \left(-\Phi+\frac{2 \pi(1-j)}{M}\right)} \\
& +\left(\frac{1}{4}-\frac{1}{4 M}+\frac{\Gamma_{1}+\Gamma_{2}}{4 \pi}\right)\left(\frac{\cos \Theta_{1}}{\sin ^{2} \Theta_{1}}-\frac{\cos \Theta_{2}}{\sin ^{2} \Theta_{2}}\right) \\
& +\frac{\Gamma_{1}-\Gamma_{2}}{4 \pi}\left(\frac{1}{\sin ^{2} \Theta_{1}}-\frac{1}{\sin ^{2} \Theta_{2}}\right) .
\end{aligned}
$$

Consequently, we have only to plot the contour map of the Hamiltonian (19) in the domain $\left(\Phi, \Theta_{1}\right)$ to obtain global dynamics of the reduced system.

Last, let us remark the meaning of the reduction in terms of the theory of dynamical systems. It follows from the symmetry (18) that the velocity field satisfies

$$
\dot{\Theta}_{2 m-1}=\dot{\Theta}_{1}, \dot{\Theta}_{2 m}=\dot{\Theta}_{2}, \dot{\Psi}_{2 m-1}=\dot{\Psi}_{1}, \dot{\Psi}_{2 m}=\dot{\Psi}_{2} \quad \text { for } m=1, \cdots, M .
$$

On the other hand, Theorem 8 shows that the eigenvectors (17) corresponding to $\lambda_{M}^{ \pm}$have the same relation, which indicates that the reduced system of the $N$ ring is equivalent to the planar phase space spanned by $\vec{\phi}_{M}^{ \pm}$. Therefore, if all the eigenvalues $\lambda_{p}^{ \pm}$for $p=1,2, \cdots, M-1$ except the simple $\lambda_{M}^{+}$are neutrally stable, that is to say the stability condition (15) for $\lambda_{M-1}^{ \pm}$

$$
\frac{N}{4}+\frac{\cos ^{2} \theta_{0}}{N}<\kappa_{1}\left(1+\cos ^{2} \theta_{0}\right)+\kappa_{2} \cos \theta_{0}
$$


holds, then the perturbed $N$-ring is subject to the reduced system. On the contrary, if some eigenvalues become unstable, the orbit starting from the neighborhood of the $N$-ring follows the reduced system only for an initial moment and eventually deviates from the system due to the existence of the unstable manifold transverse to the planar phase space of the reduced system.

\section{Global dynamics of the reduced system}

\subsection{The 4-ring at the equator}

We consider the motion of the reduced system when the 4-ring is placed at the equator, i.e. $N=4$ and $\theta_{0}=\frac{\pi}{2}$. It follows from (24) that we have $\Theta_{1}+\Theta_{2}=\frac{\pi}{2}$. The Hamiltonian (19) and the equations (20) and (25) are reduced to

$$
\begin{aligned}
H= & -\frac{\pi}{8} \log \left(1-\cos ^{2} \Theta_{1}+\sin ^{2} \Theta_{1}\right)-\frac{\pi}{8} \log \left(1+\cos ^{2} \Theta_{1}-\sin ^{2} \Theta_{1} \cos (\Phi-\pi)\right) \\
& -\frac{\pi}{8} \log \left(1+\cos ^{2} \Theta_{1}-\sin ^{2} \Theta_{1} \cos \Phi\right)-\frac{\Gamma_{1}+\Gamma_{2}}{4} \log \left(1-\cos ^{2} \Theta_{1}\right) \\
\dot{\Theta}_{1}= & -\frac{1}{4} \frac{\sin ^{3} \Theta_{1} \sin \Phi \cos \Phi}{\left(1+\cos ^{2} \Theta_{1}\right)^{2}-\sin ^{4} \Theta_{1} \cos ^{2} \Phi} \equiv F_{1}\left(\Theta_{1}, \Phi\right) \\
\dot{\Phi}= & -\frac{\cos \Theta_{1}}{4}\left(\frac{1+\cos \Phi}{1+\cos ^{2} \Theta_{1}-\sin ^{2} \Theta_{1} \cos \Phi}-\frac{1-\cos \Phi}{1+\cos ^{2} \Theta_{1}+\sin ^{2} \Theta_{1} \cos \Phi}\right) \\
& +\left(\frac{1}{4}+\frac{\Gamma_{1}+\Gamma_{1}}{2 \pi}\right) \frac{\cos \Theta_{1}}{\sin ^{2} \Theta_{1}} \\
\equiv & F_{2}\left(\Theta_{1}, \Phi\right) .
\end{aligned}
$$

First, we obtain the fixed points of the reduced equations.

Proposition 9 The fixed points of the equations (29) and (30) are given as follows:

(1) $\sin \Phi=0$ and $\tan ^{2} \Theta_{1}=4 \kappa_{1}-3$; (collinear fixed points),

(2) $\cos \Phi=0$ and $\tan ^{2} \Theta_{1}=\frac{8 \kappa_{1}-6}{5-4 \kappa_{1}} ;$ (staggered fixed points),

(3) $\cos \Phi=0$ and $\Theta_{1}=\frac{\pi}{2}$; (4-ring fixed points).

Proof: It follows from $\dot{\Theta}_{1}=0$ that we obtain either $\sin \Phi=0$ or $\cos \Phi=0$. Substituting $\cos \Phi=1$ to (30), we have

$$
\left(4 \kappa_{1}-3\right) \frac{\cos \Theta_{1}}{\sin ^{2} \Theta_{1}}-\frac{1}{\cos \Theta_{1}}=0 .
$$

Hence, we have $\tan ^{2} \Theta_{1}=4 \kappa_{1}-3$. On the other hand, when $\cos \Phi=0$, the equation (30) becomes

$$
\left(\kappa_{1}-\frac{3}{4}\right) \frac{\cos \Theta_{1}}{\sin ^{2} \Theta_{1}}-\frac{\cos \Theta_{1}}{2} \frac{1}{1+\cos ^{2} \Theta_{1}}=0,
$$

which yields $\cos \Theta_{1}=0$ or $\tan ^{2} \Theta_{1}=\frac{8 \kappa_{1}-6}{5-4 \kappa_{1}}$.

The following proposition gives existence and linear stability of the fixed points.

Proposition 10 The collinear fixed points exist for $\kappa_{1}>\frac{3}{4}$ and they are hyperbolic. The staggered fixed points exist for $\frac{3}{4}<\kappa_{1}<\frac{5}{4}$ and they are elliptic. The 4-ring fixed points exist for all $\kappa_{1}$ and they are hyperbolic for $\kappa_{1}<\frac{5}{4}$ and elliptic $\kappa_{1}>\frac{5}{4}$. 
Let us remember the stability of the 4-ring in Corollary 7; When $N=4$ and $\theta_{0}=\frac{\pi}{2}$, it follows from (16) that the 4-ring is stable if $\kappa_{1}>\frac{5}{4}$, and unstable if $\kappa_{1}<\frac{5}{4}$, which agrees with the stability result in Proposition 10.

Proof: Since $\tan ^{2} \Theta_{1}$ is positive, the collinear fixed points and the staggered fixed points exist if $\kappa_{1}>\frac{3}{4}$ and $\frac{3}{4}<\kappa_{1}<\frac{5}{4}$, respectively.

As for the linear stability of the fixed points, it is suffice to compute the eigenvalues of the following linearized matrix of (29) and (30),

$$
\left(\begin{array}{ll}
\frac{\partial F_{1}}{\partial \Theta_{1}} & \frac{\partial F_{1}}{\partial \Phi} \\
\frac{\partial F_{2}}{\partial \Theta_{1}} & \frac{\partial F_{2}}{\partial \Phi}
\end{array}\right),
$$

in which

$$
\begin{aligned}
\frac{\partial F_{1}}{\partial \Theta_{1}}= & -\frac{\sin \Phi \cos \Phi}{4} \frac{\partial}{\partial \Theta_{1}}\left(\frac{\sin ^{3} \Theta_{1}}{\left(1+\cos ^{2} \Theta_{1}\right)^{2}-\sin ^{4} \Theta_{1} \cos ^{2} \Phi}\right), \\
\frac{\partial F_{1}}{\partial \Phi}= & -\frac{\sin ^{3} \Theta_{1}}{4} \frac{\left(\cos ^{2} \Phi-\sin ^{2} \Phi\right)\left(1+\cos ^{2} \Theta_{1}\right)-\sin ^{4} \Theta_{1} \cos ^{2} \Phi}{\left(\left(1+\cos ^{2} \Theta_{1}\right)^{2}-\sin ^{4} \Theta_{1} \cos ^{2} \Phi\right)^{2}}, \\
\frac{\partial F_{2}}{\partial \Theta_{1}}= & -\left(\kappa_{1}-\frac{3}{4}\right) \frac{1+\cos ^{2} \Theta_{1}}{\sin ^{3} \Theta_{1}}+\frac{\sin \Theta_{1}}{2}\left(\frac{1+\cos ^{2} \Theta_{1}+\sin ^{2} \Theta_{1} \cos \Phi}{\left(1+\cos ^{2} \Theta_{1}\right)^{2}-\sin ^{4} \Theta_{1} \cos ^{2} \Phi}\right) \\
& -\frac{\cos ^{2} \Theta_{1}}{2}\left(\frac{\sin \Theta_{1}(1+\cos \Phi)^{2}}{\left(1+\cos ^{2} \Theta_{1}-\sin ^{2} \Theta_{1} \cos \Phi\right)^{2}}+\frac{\sin \Theta_{1}(1-\cos \Phi)^{2}}{\left(1+\cos ^{2} \Theta_{1}+\sin ^{2} \Theta_{1} \cos \Phi\right)^{2}}\right), \\
\frac{\partial F_{2}}{\partial \Phi}= & -\frac{\cos \Theta_{1}}{2}\left(\frac{-\sin ^{2} \Phi\left(1+\cos ^{2} \Theta_{1}\right)}{\left(1+\cos ^{2} \Theta_{1}-\sin ^{2} \Theta_{1} \cos \Phi\right)^{2}}+\frac{\sin \Phi\left(1+\cos ^{2} \Theta_{1}\right)}{\left(1+\cos ^{2} \Theta_{1}+\sin ^{2} \Theta_{1} \cos \Phi\right)^{2}}\right) .
\end{aligned}
$$

For the collinear fixed points, since $\sin \Phi=0$ and $\tan ^{2} \Theta_{1}=4 \kappa_{1}-3$, we have

$$
\frac{\partial F_{1}}{\partial \Theta_{1}}=\frac{\partial F_{2}}{\partial \Phi}=0, \quad \frac{\partial F_{1}}{\partial \Phi}=-\frac{4 \kappa_{1}-3}{16} \sqrt{\frac{4 \kappa_{1}-3}{4 \kappa_{1}-2}}, \quad \frac{\partial F_{2}}{\partial \Theta_{1}}=-\left(2 \kappa_{1}-1\right) \sqrt{\frac{4 \kappa_{1}-2}{4 \kappa_{1}-3}} .
$$

Hence, since $\kappa_{1}>\frac{3}{4}$, the eigenvalue $\sigma$ satisfies

$$
\sigma^{2}=\frac{1}{16}\left(2 \kappa_{1}-1\right)\left(4 \kappa_{1}-3\right)>0,
$$

which shows that the collinear fixed points are hyperbolic.

For the staggered fixed points, since $\cos \Phi=0$ and $\tan ^{2} \Theta_{1}=\frac{8 \kappa_{1}-3}{5-4 \kappa_{1}}$, we have

$$
\begin{aligned}
& \frac{\partial F_{1}}{\partial \Theta_{1}}=\frac{\partial F_{2}}{\partial \Phi}=0, \frac{\partial F_{1}}{\partial \Phi}=\frac{\left(4 \kappa_{1}-3\right)\left(4 \kappa_{1}-1\right)}{32} \sqrt{\frac{8 \kappa_{1}-6}{4 \kappa_{1}-1}}, \\
& \frac{\partial F_{2}}{\partial \Theta_{1}}=-\frac{1}{2} \sqrt{\frac{4 \kappa_{1}-1}{8 \kappa_{1}-6}}+\frac{\left(4 \kappa_{1}-3\right)\left(4 \kappa_{1}-1\right)}{16} \sqrt{\frac{8 \kappa_{1}-6}{4 \kappa_{1}-1}} .
\end{aligned}
$$

Therefore, the eigenvalue $\sigma$ is represented by

$$
\sigma^{2}=\frac{1}{256}\left(4 \kappa_{1}-1\right)^{2}\left(4 \kappa_{1}-3\right)\left(4 \kappa_{1}-5\right)<0,
$$

which indicates that the staggered fixed points are elliptic due to $\frac{3}{4}<\kappa_{1}<\frac{5}{4}$.

For the 4-ring fixed points, since $\cos \Phi=0$ and $\Theta_{1}=\frac{\pi}{2}$, the eigenvalue $\sigma$ is given by

$$
\sigma^{2}=\frac{1}{16}\left(5-4 \kappa_{1}\right)
$$

Hence, the 4-ring fixed points are elliptic for $\kappa_{1}>\frac{5}{4}$ and hyperbolic for $\kappa_{1}<\frac{5}{4}$. 
In what follows, we plot the contour maps of the Hamiltonian (28) in the domain $\left(\Phi, \Theta_{1}\right) \in[0,2 \pi] \times[0, \pi]$ to see global dynamics of the reduced system. Since the number of the fixed points and their stability change at $\kappa_{1}=\frac{3}{4}$ and $\kappa_{1}=\frac{5}{4}$, we plot the contour maps between them, i.e. $\kappa_{1}=1.4,1.1,0.9$, and 0.6 in Figure 2. When $\kappa_{1}=1.4$, there exist the hyperbolic collinear fixed points and the elliptic 4-ring fixed points given in Proposition 9 and Proposition 10. The collinear fixed points are connected by heteroclinic orbits. When we perturb the elliptic 4-ring fixed point slightly, the solution rotates around the fixed point. For $\kappa_{1}=1.1$, the 4-ring fixed points become unstable and the elliptic staggered fixed points develop. While the heteroclinic orbits connecting the hyperbolic collinear fixed points still remain, new homoclinic orbits of the 4-ring fixed point surrounding the staggered fixed point emerge. Then, the perturbed 4-ring returns to its original position periodically, although the 4-ring fixed point is unstable.

The global dynamics of the perturbed 4-ring changes when $\kappa_{1}=0.9$, although the number of the fixed points and their stability are unchanged. We observe new heteroclinic orbits between the hyperbolic 4-ring fixed points, and heteroclinic orbits between the hyperbolic collinear fixed points enclosing the staggered fixed point. Thus, there are two possible periodic orbits starting from the neighborhood of the 4-ring fixed point; The vortex point returns periodically to its initial position after passing near the next 4-ring fixed point, or it keeps moving in the longitudinal direction. When $\kappa_{1}=0.6$, the collinear and the staggered fixed points disappear, and only the hyperbolic 4-ring fixed points and the heteroclinic orbits between them survive. Hence, the global motion of the perturbed 4-ring fixed point is the same as in the previous case.

Figure 3 shows the periodic orbits of the pair of the two vortex points $\left(\Theta_{1}, \Psi_{1}\right)$ and $\left(\Theta_{2}, \Psi_{2}\right)$ on the sphere for various $\kappa_{1}$, each of which corresponds to a contour line of the perturbed 4-ring in Figure 2. The rest of the vortex points evolve likewise on the back of the sphere according to the symmetry (18). Since we have $\Theta_{1}+\Theta_{2}=\frac{\pi}{2}$, $\Theta_{1}$ and $\Theta_{2}$ are symmetric with respect to $\frac{\pi}{2}$. First, when $\kappa_{1}=1.4$, each of the vortex points rotates around the 4-ring fixed point, which we call linearly periodic. Next, although the 4-ring fixed point is linearly unstable when $\kappa_{1}=1.1$, the perturbed 4-ring still goes around the 4-ring fixed point periodically. We refer to the orbit as a nonlinearly periodic orbit in order to distinguish it from the linearly periodic orbit. Last, for $\kappa_{1}=0.9$, two types of periodic orbits are possible; The first one is a swing-by orbit, in which the two vortex points return to the initial locations after switching their relative position. The second one is a revolving orbit, in which the one vortex point moves eastwards bouncing on the equator and the other moves similarly in the opposite direction.

The reason why the global dynamics changes between $\kappa_{1}=1.1$ and $\kappa_{1}=0.9$ is that the separatrices exchange their connection. Namely, while the separatrices consist of the heteroclinic orbits between the collinear fixed points and the homoclinic orbits of the 4-ring fixed point for $\kappa_{1}=1.1$, we observe the other heteroclinic orbits between the collinear fixed points and the heteroclinic orbits between the 4-ring fixed points for $\kappa_{1}=0.9$. It means that the heteroclinic orbits and the homoclinic orbits for $\kappa_{1}=1.1$ must agree with each other at some marginal value of $\kappa_{1} \in[0.9,1.1]$. When the interchange of the separatrices occurs, the value of the Hamiltonian (28) at the collinear fixed points equals to that of the 4-ring fixed points. Hence, we have

$$
\frac{1}{8} \log \left(\frac{4 \kappa_{1}-3}{4 \kappa_{1}-1}\right)-\frac{1}{8} \log \left(2 \kappa_{1}-1\right)+\frac{1}{2}\left(\kappa_{1}-1\right) \log \left(\frac{4 \kappa_{1}-3}{4 \kappa_{1}-1}\right)=0,
$$

which yields $\kappa_{1}=1$. Figure 4 shows the contour plot of the Hamiltonian for $\kappa_{1}=$ 1 , which indicates that the perturbed 4-ring evolves along either the nonlinearly periodic orbit or the swing-by orbit. 
(a)

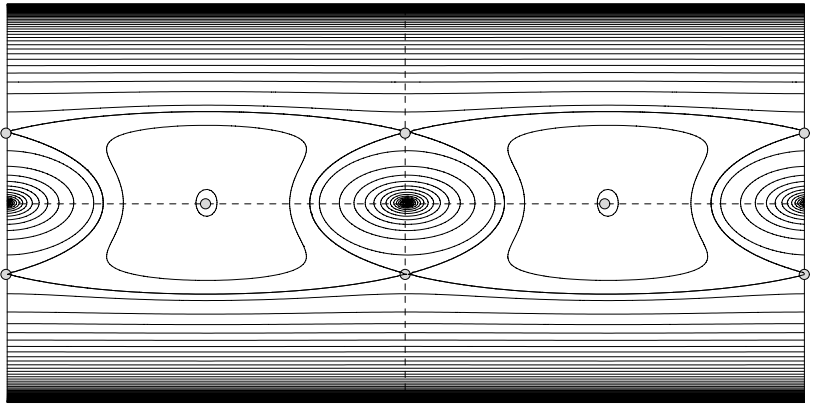

(b)

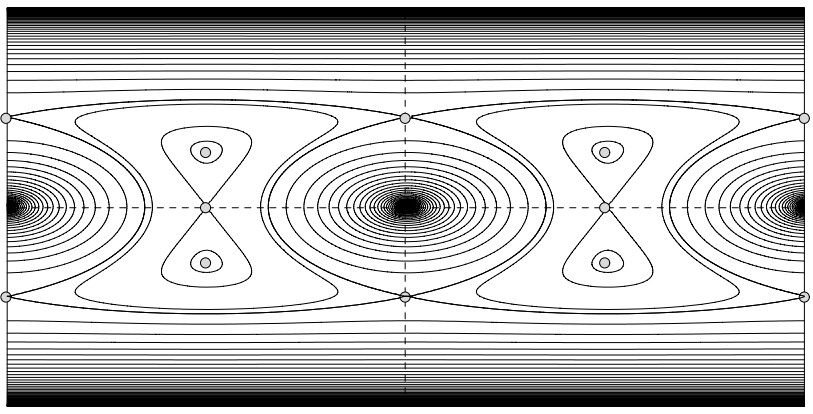

(c)

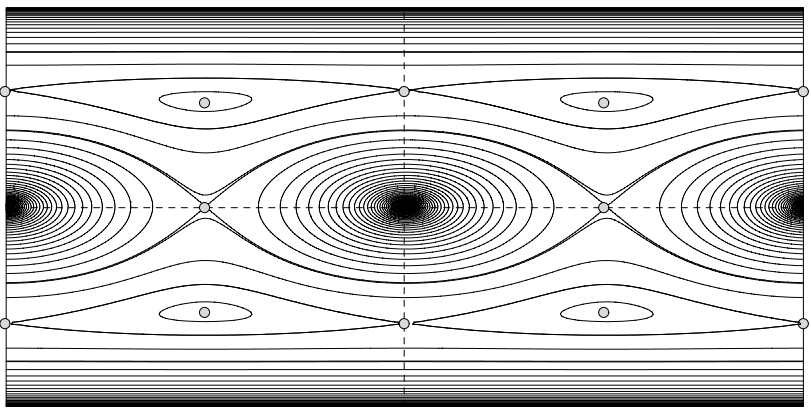

(d)

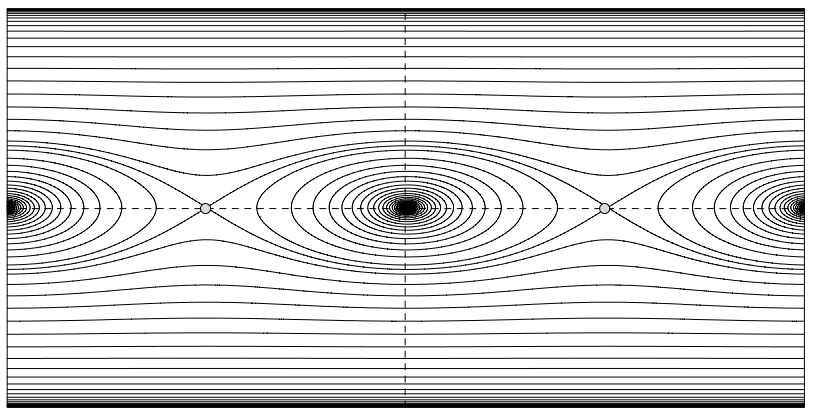

Figure 2: Contour maps of the Hamiltonian for the 4-ring at the equator plotted in the domain $\left(\Phi, \Theta_{1}\right) \in[0,2 \pi] \times[0, \pi]$. (a) $\kappa_{1}=1.4$, (b) $\kappa_{1}=1.1$, (c) $\kappa_{1}=0.9$, and (d) $\kappa_{1}=0.6$. 
(a) linearly periodic orbit

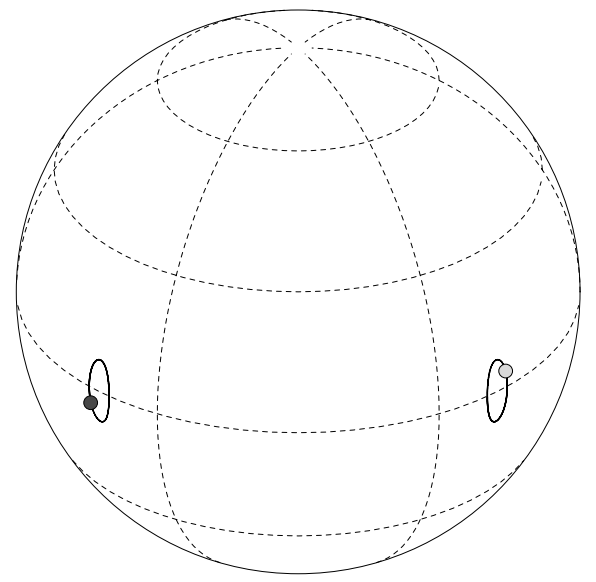

(b) nonlinearly periodic orbit

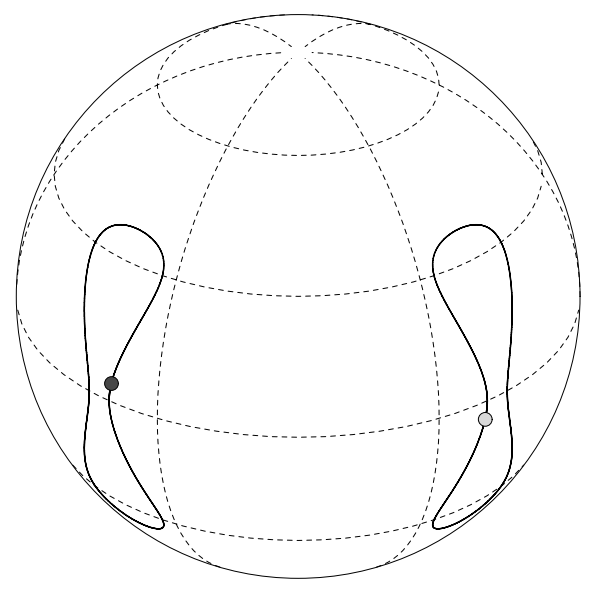

(c) swing-by orbit

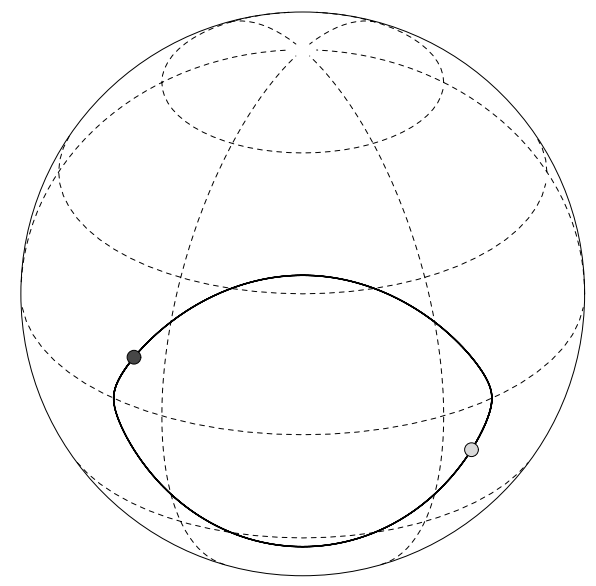

(d) revolving orbit

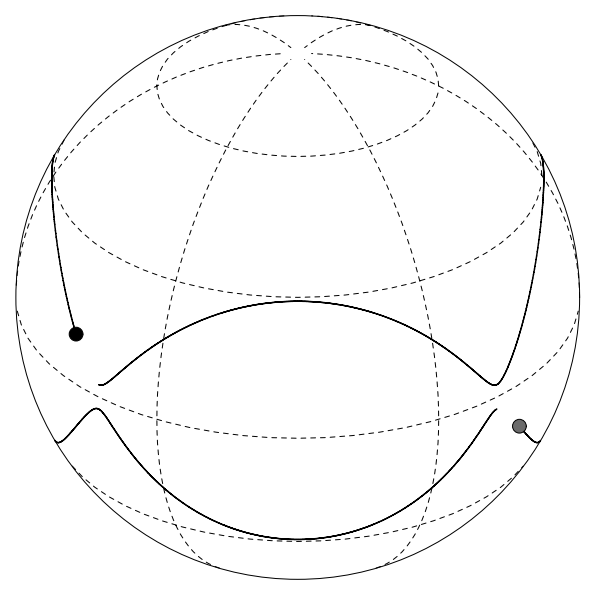

Figure 3: Four orbits of the perturbed 4-ring fixed points at the equator observed typically in the reduced system. The pair of the two vortex points $\left(\Theta_{1}, \Psi_{1}\right)$ and $\left(\Theta_{2}, \Psi_{2}\right)$ are plotted. The other two vortex points evolve likewise on the back of the sphere due to the symmetry (18). (a) Linearly periodic orbit $\left(\kappa_{1}=1.4\right)$, (b) nonlinearly periodic orbit $\left(\kappa_{1}=1.1\right)$, (c) swing-by orbit $\left(\kappa_{1}=0.9\right)$, and (d) revolving orbit $\left(\kappa_{1}=0.9\right)$. 


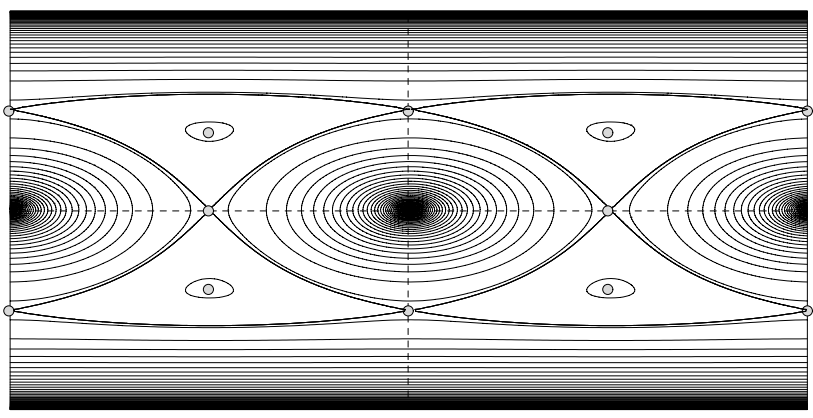

Figure 4: Contour map of the Hamiltonian for the 4-ring at the equator for $\kappa_{1}=1$, across which the separatrices interchange the connection.

Thus, organizing the observation and the propositions above, we describe the dynamics of the reduced system.

Theorem 11 The dynamics of the reduced 4-ring at the equator is classified into the following five types.

\begin{tabular}{|c|c|c|c|}
\hline Type & $\kappa_{1}$ & fixed points & perturbed 4-ring \\
\hline$I$ & $\frac{5}{4}<\kappa_{1}$ & $\begin{array}{c}\text { hyperbolic collinear } \\
\text { elliptic 4-ring }\end{array}$ & linearly periodic \\
\hline$I I$ & $1<\kappa_{1}<\frac{5}{4}$ & $\begin{array}{l}\text { hyperbolic collinear } \\
\text { hyperbolic 4-ring } \\
\text { elliptic staggered }\end{array}$ & nonlinearly periodic \\
\hline III & $\kappa_{1}=1$ & $\begin{array}{c}\text { hyperbolic collinear } \\
\text { hyperbolic 4-ring } \\
\text { elliptic staggered }\end{array}$ & $\begin{array}{c}\text { nonlinearly periodic } \\
\text { swing-by }\end{array}$ \\
\hline$I V$ & $\frac{3}{4}<\kappa_{1}<1$ & $\begin{array}{c}\text { hyperbolic collinear } \\
\text { hyperbolic 4-ring } \\
\text { elliptic staggered }\end{array}$ & $\begin{array}{l}\text { swing-by } \\
\text { revolving }\end{array}$ \\
\hline$V$ & $\kappa_{1}<\frac{3}{4}$ & hyperbolic 4-ring & $\begin{array}{l}\text { swing-by } \\
\text { revolving }\end{array}$ \\
\hline
\end{tabular}

As we have discussed in the previous section, if the eigenvalues $\lambda_{1}^{ \pm}$for the 4-ring are neutrally stable, i.e. $\kappa_{1}>1$ from (27), then the linearly periodic orbits and the nonlinearly periodic orbits are stable in the sense that the perturbed 4-ring evolves in the neighborhood of the periodic orbits for all the time. On the contrary, if $\lambda_{1}^{+}$becomes unstable, the perturbed 4-ring evolves unstably after behaving like a swing-by orbit or a revolving orbit for a short time, because it eventually leaves the reduced system along the unstable manifold corresponding to $\lambda_{1}^{+}$. Let us finally note that the transition of the global dynamics and the stability of the $\lambda_{1}^{ \pm}$change at the same value $\kappa_{1}=1$ in this example by a coincidence. However, they occur independently in general, as we see in the next subsection.

\subsection{The 4-ring at the line of latitude $\theta_{0}=\frac{\pi}{4}$}

We consider the global motion of the perturbed 4-ring at the line of latitude $\theta_{0}=\frac{\pi}{4}$. In general, it is difficult to obtain the fixed points of (20), (21), (22), and (23) 
mathematically for $\theta_{0} \neq \frac{\pi}{2}$ and so we compute them numerically.

In order to describe the global dynamics, let us remember the stability condition (15) for $\theta_{0}=\frac{\pi}{4}$ and $N=4$; The eigenvalues $\lambda_{2}^{ \pm}$are neutrally stable if $\kappa>\frac{11}{8}=$ 1.375 , and the eigenvalues $\lambda_{1}^{ \pm}$are neutrally stable if $\kappa>\frac{9}{8}=1.125$, in which $\kappa \equiv \kappa_{1}\left(1+\cos ^{2} \theta_{0}\right)+\kappa_{2} \cos \theta_{0}=\frac{3}{2} \kappa_{1}+\frac{\sqrt{2}}{2} \kappa_{2}$ is the parameter that determines the stability of the eigenvalues appearing in Theorem 6 . We also note that the variable $\Theta_{1}$ moves in the range of

$$
2 \cos \theta_{0}-1 \leq \cos \Theta_{1} \leq 1
$$

due to (24).

Figure 5 shows the contour maps of the Hamiltonian for $\kappa=1.5,1.3,1.15,1$, when the strength of the north pole vortex equals to that of the south pole vortex, i.e. $\kappa_{2}=0$. Each of the contour maps is topologically the same as that in Figure 2. Consequently, as the parameter $\kappa$ decreases, we observe the same transition of global dynamics from the type $\mathrm{I}$ to $\mathrm{V}$ described in Theorem 11. The interchange of the separatrices and the transition from the type IV to $\mathrm{V}$ occur at $\kappa=1.1935$ and $\kappa=1.125$, respectively.

Since we have $\cos \Theta_{1}+\cos \Theta_{2}=\sqrt{2}$ in this case, the contour line of the perturbed 4-ring disagrees with the actual orbit of the vortex point on the sphere. We plot the periodic orbits of the perturbed 4-ring in Figure 6, when they are viewed from the north. Figure 6 (a) shows that the orbit for $\kappa=1.5$ is linearly periodic, which rotates around the 4-ring fixed point. When $\kappa=1.3$, it becomes nonlinearly periodic. On the other hand, for $\kappa=1.15$, the global motion of the perturbed 4-ring changes due to the interchange of the separatrices. Figure 6 (c) and (d) show the swing-by orbit and the revolving orbit, respectively.

Since the interchange of the separatrices happens while $\lambda_{1}^{ \pm}$are still neutrally stable in this case, not only the linearly and nonlinearly periodic orbits but also the swing-by and the revolving orbits are stable. That is to say, the perturbed 4-ring follows a linearly periodic orbit for $\kappa>1.375$, a nonlinearly periodic orbit for $1.1935<\kappa<1.375$, and either a swing-by orbit or a revolving orbit for $1.125<$ $\kappa<1.1935$.

Finally, we show the contour maps of the Hamiltonian when we change $\kappa_{1}$ with keeping $\kappa_{2}=\pi$ in Figure 7 . It indicates that the same transition of the global dynamics is observed as well for the other $\kappa_{2}$. The type III is observed when $\kappa=1.1674$ and the transition from the type IV to $\mathrm{V}$ happens at $\kappa=1.082$. They are different from the case of $\kappa_{2}=0$.

\subsection{The $N$-rings for $N=6$ and 8}

We plot the contour maps of the Hamiltonian for the 6-ring and the 8-ring at the equator $\theta_{0}=\frac{\pi}{2}$ in Figure 8 and 9, respectively. Each figure shows that the same transition of global dynamics of the perturbed $N$-ring occurs through the interchange of the separatrices. It implies that the transition of the global dynamics is observed universally regardless of the number of vortex points.

\section{Conclusion}

We have investigated the linear stability and the global dynamics of the $N$-ring in which the identical vortex points are equally placed along the line of latitude $\theta_{0}$ on the sphere with the pole vortices, when the number of vortex points is even, i.e. $N=2 M$. 
(a)

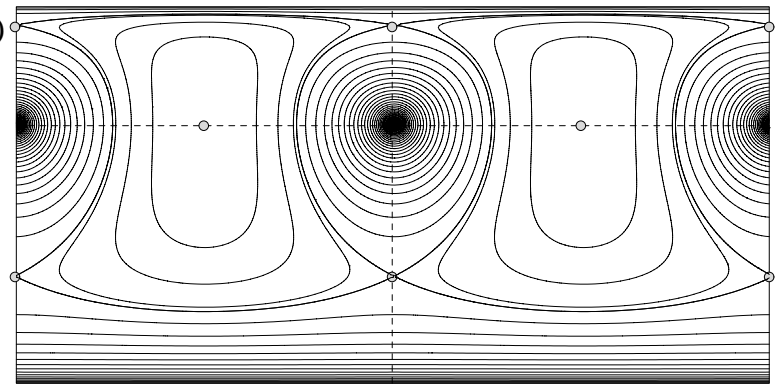

(b)

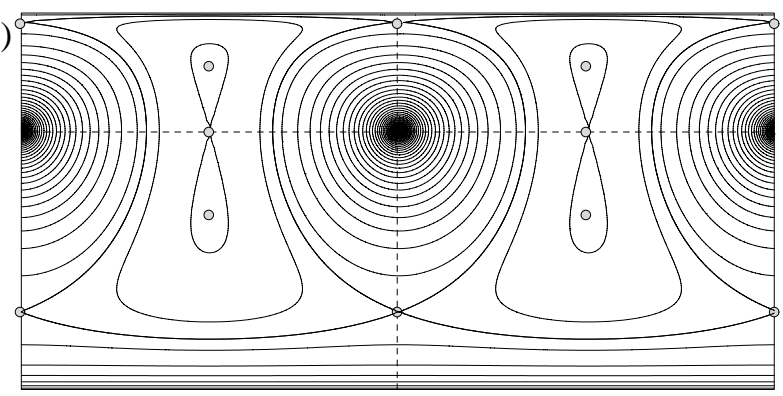

(c)

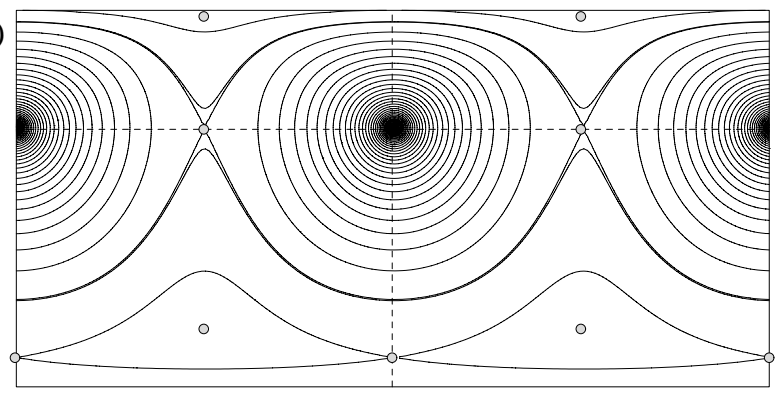

(d)

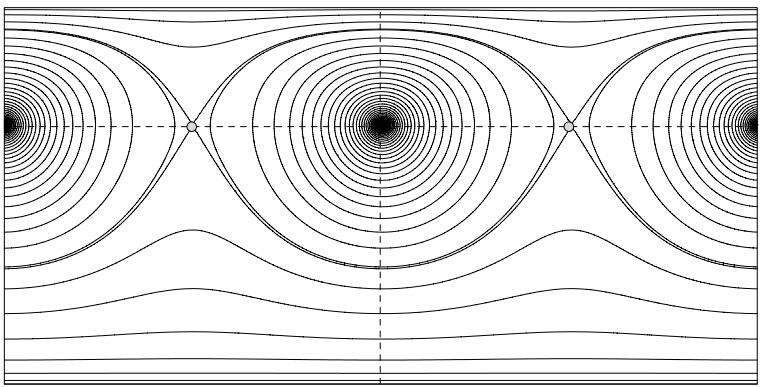

Figure 5: Contour maps of the Hamiltonian for the 4-ring at the line of latitude $\theta_{0}=\frac{\pi}{4}$ in the domain $\left(\Phi, \Theta_{1}\right) \in[0,2 \pi] \times\left[0, \Theta_{c}\right]$, in which $\Theta_{c}$ satisfies $\cos \Theta_{c}=$ $2 \cos \theta_{0}-1$. The parameter $\kappa_{2}$ is fixed to zero and we change $\kappa_{1}$. (a) $\kappa=1.5$, (b) $\kappa=1.3$, (c) $\kappa=1.15$, and (d) $\kappa=1.0$, where $\kappa=\frac{3}{2} \kappa_{1}+\frac{\sqrt{2}}{2} \kappa_{2}$. The interchange of the separatrices from (b) to (c) occurs at $\kappa=1.1935$. 
(a) periodic orbit

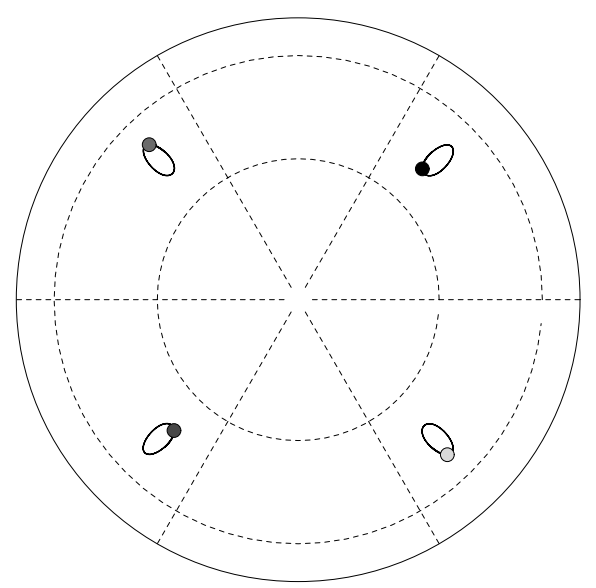

(b) nonlinearly periodic orbit

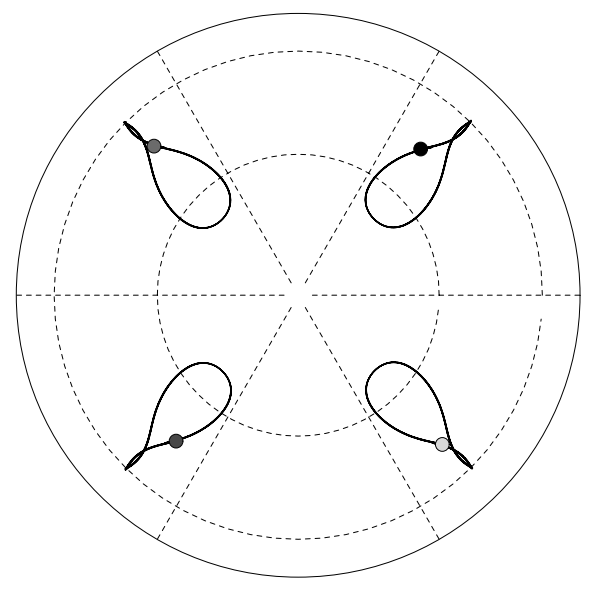

(c) swing-by orbit

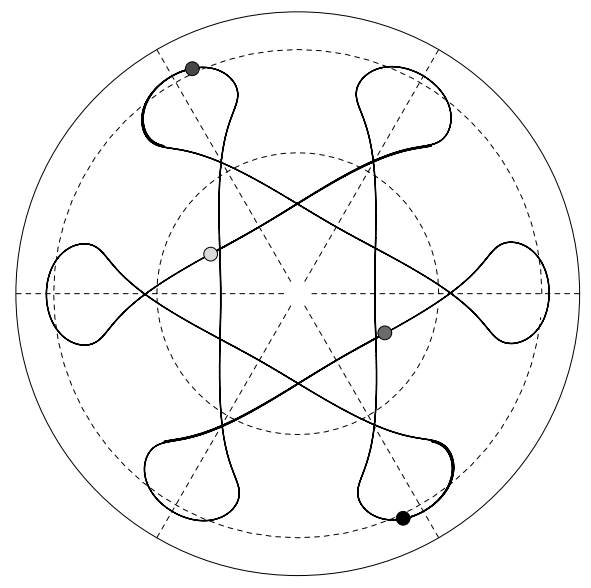

(d) revolving orbit

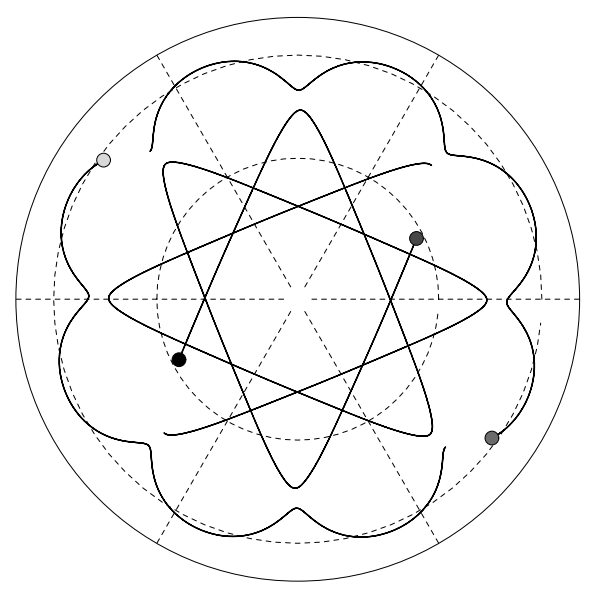

Figure 6: Four periodic orbits of the perturbed 4-ring fixed point at the line of latitude $\theta_{0}=\frac{\pi}{4}$ observed typically in the reduced system, which are viewed from the north. We plot the orbits of all the vortex points on the sphere. (a) Linearly periodic orbit $(\kappa=1.5)$, (b) nonlinearly periodic orbit $\left(\kappa_{1}=1.3\right)$, (c) swing-by orbit $(\kappa=1.1)$, and $(\mathrm{d})$ revolving orbit $\left(\kappa_{1}=1.1\right)$. 
(a)

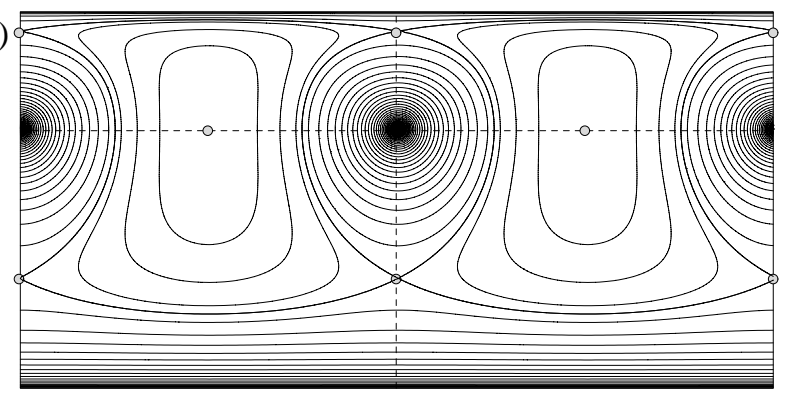

(b)

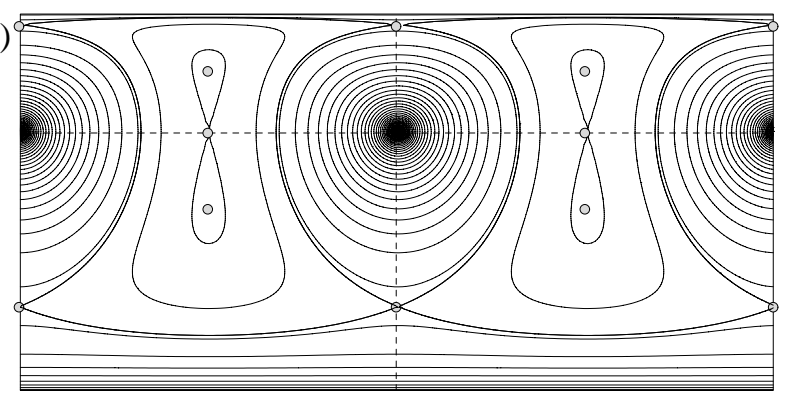

(c)

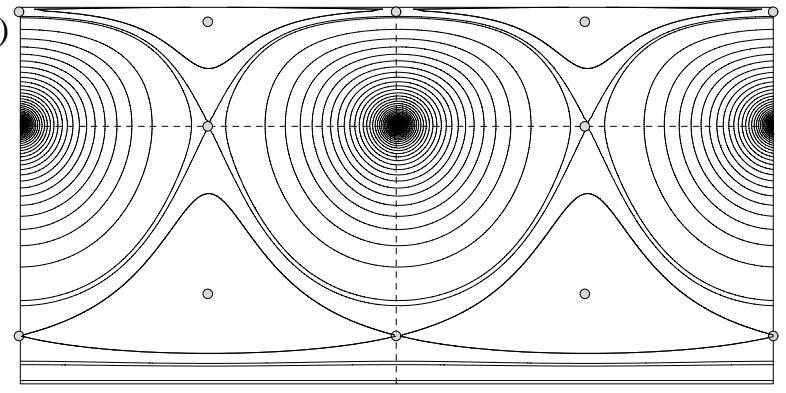

(d)

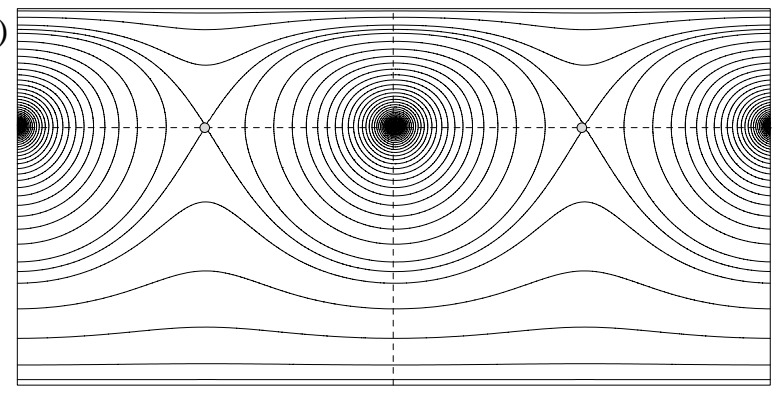

Figure 7: Contour maps of the Hamiltonian for the 4-ring at the line of latitude $\theta_{0}=\frac{\pi}{4}$ in the same domain of $\left(\Phi, \Theta_{1}\right)$ as in Figure 5. The parameter $\kappa_{2}$ is fixed to $\pi$ and we change $\kappa_{1}$. (a) $\kappa=1.5$, (b) $\kappa=1.3$, (c) $\kappa=1.15$, and (d) $\kappa=1$. The separatrices exchange their connection at $\kappa=1.1674$. 
(a)

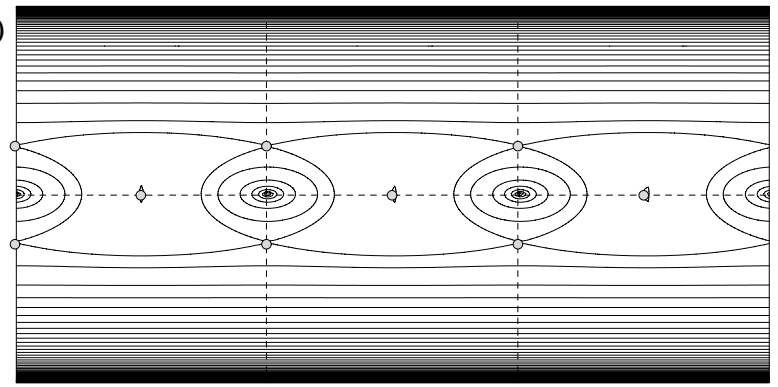

(b)

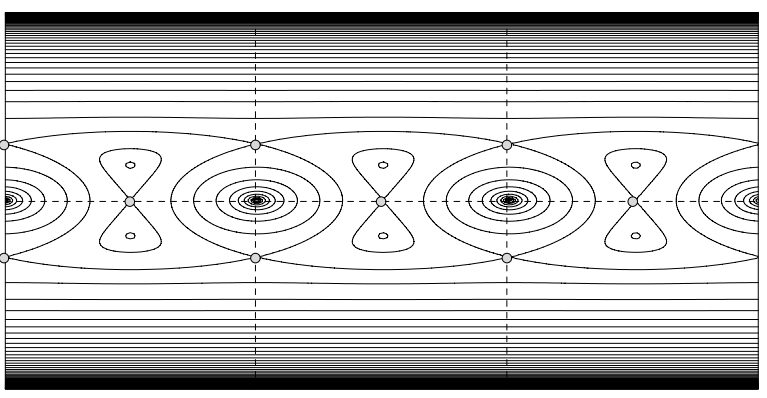

(c)

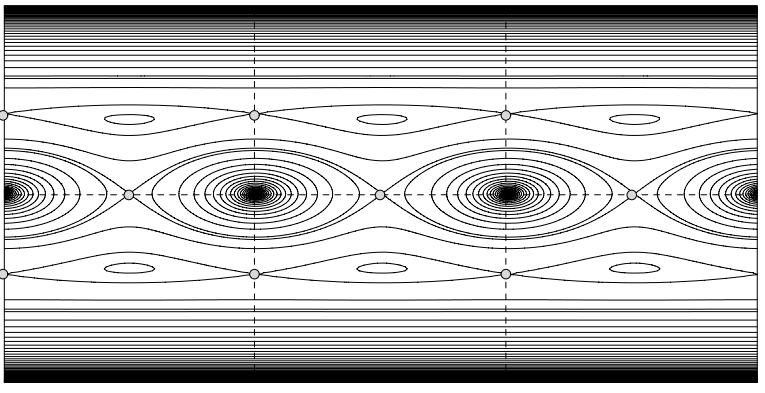

(d)

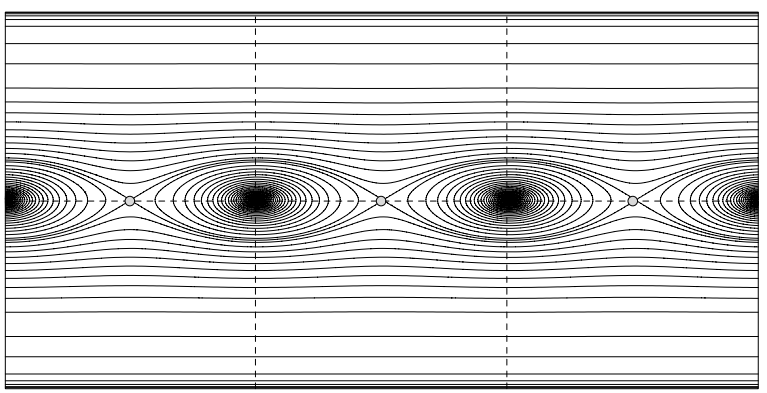

Figure 8: Contour maps of the Hamiltonian for the 6-ring at the equator $\theta_{0}=\frac{\pi}{2}$ for various $\kappa=\kappa_{1}$. Note that the staggered fixed points are not displayed in the figure. (a) $\kappa=1.7$, (b) $\kappa=1.4$, (c) $\kappa=1$, and (d) $\kappa=0.7$. 
(a)

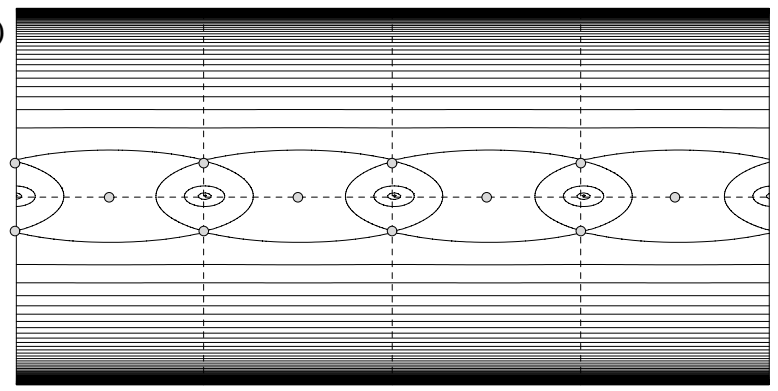

(b)

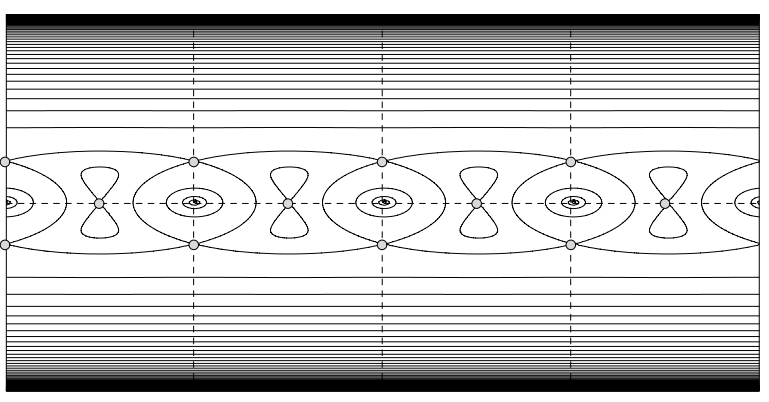

(c)

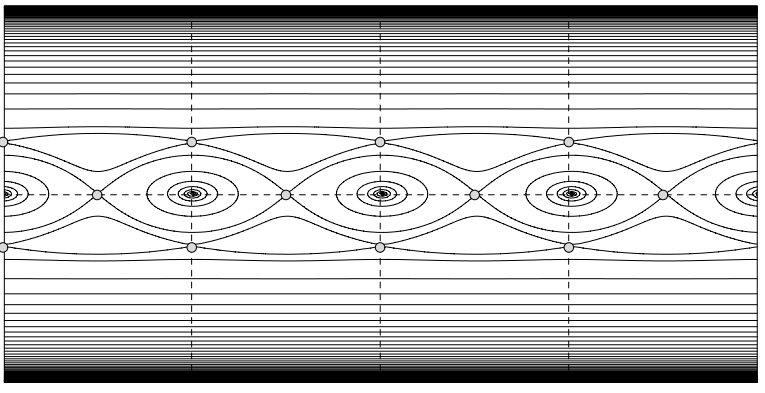

(d)

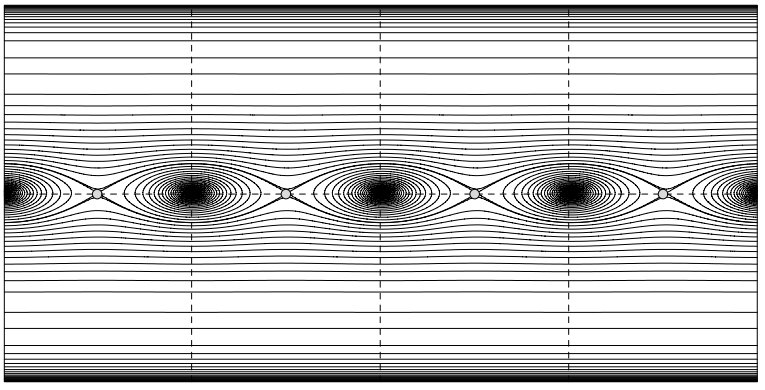

Figure 9: Contour maps of the Hamiltonian for the 8-ring at the equator $\theta_{0}=\frac{\pi}{2}$ for various $\kappa=\kappa_{1}$. The staggered fixed points are not displayed in the figure. (a) $\kappa=2.2$, (b) $\kappa=1.8$, (c) $\kappa=1.4$, and (d) $\kappa=0.7$. 
First, we give explicitly all the eigenvalues and the eigenvectors of the linearized equations for the $N$-ring in Theorem 4 and Theorem 8 . There exist $M+1$ eigenvalues, denoted by $\lambda_{p}^{ \pm}$for $p=0,1, \cdots, M$. The eigenvalues $\lambda_{0}^{ \pm}$are zero and their eigenvectors are equivalent to the rotational vectors in the longitudinal directions. The eigenvalues $\lambda_{p}^{ \pm}$for $p=1, \cdots, M-1$ are double and each of them has two linearly independent eigenvectors that are complex conjugate with each other. Only the eigenvalues $\lambda_{M}^{ \pm}$are simple and the eigenvectors have the alternate symmetry that pairs the adjoining two vortex points together.

The stability of the eigenvalues is determined by the number of vortex points, the strengths of the pole vortices and the position of the $N$-ring. The exact stability condition is given in Theorem 6 , which shows that the eigenvalues satisfy the following order,

$$
\left(\lambda_{1}^{ \pm}\right)^{2}<\left(\lambda_{2}^{ \pm}\right)^{2}<\cdots<\left(\lambda_{M}^{ \pm}\right)^{2} .
$$

Accordingly, if $\left(\lambda_{m-1}^{ \pm}\right)^{2}<0<\left(\lambda_{m}^{ \pm}\right)^{2}$ holds for some $m$, then $\lambda_{j}^{+}$is unstable and $\lambda_{j}^{-}$is stable for all $j \geq m$, while $\lambda_{j}^{ \pm}$become neutrally stable for $j<m$. It indicates that the stability of the $N$-ring is determined by that of the largest eigenvalues $\lambda_{M}^{ \pm}$. The condition is provided in Corollary 7 .

Next, we reduce the equations of the $N$-vortex points to those for the pair of two vortex points by assuming the alternately pairing symmetry and then we consider the global dynamics of the reduced system; As a result, we observe the following transition of global dynamics with the decrease of the parameter that determines the linear stability of the eigenvalues.

Type I: There are hyperbolic collinear fixed points connected by heteroclinic orbits, and elliptic $N$-ring fixed points. Then, since the $N$-ring fixed point is neutrally stable, the perturbed $N$-ring follows a linearly periodic orbit rotating around the $N$-ring fixed point.

Type II: While the hyperbolic collinear fixed points and the heteroclinic orbits between them remain, the $N$-ring fixed points become unstable and elliptic staggered fixed points appear. Homoclinic orbits of the $N$-ring fixed point surrounding the staggered fixed point emerge. Then, although the $N$-ring fixed point is linearly unstable, the motion of the perturbed $N$-ring becomes periodic, which we call nonlinearly periodic.

Type III: While the number and the stability of the fixed points are unchanged, heteroclinic orbits between the hyperbolic collinear fixed points and the hyperbolic $N$-ring fixed points emerge. The motion of the perturbed $N$-ring becomes either the nonlinearly periodic solution, or the swing-by solution in which the pair of the vortex points returns to the original configuration after switching their relative position.

Type IV: There are heteroclinic orbits between the hyperbolic collinear fixed points enclosing the elliptic staggered fixed point, and heteroclinic orbits between the hyperbolic $N$-ring fixed points. When we perturb the $N$-ring, it moves along either the swing-by orbit, or the revolving orbit in which the pairing two vortex points rotate in the mutually opposite longitudinal directions.

Type V: The hyperbolic fixed points and the elliptic staggered fixed points disappear. The heteroclinic orbits between the $N$-ring fixed points are left. The dynamics of the perturbed $N$-ring is the same as in the type IV. 
The interchange of the separatrices occurs at the type III, which results in the qualitative change of the global periodic motion of the perturbed $N$-ring from the nonlinearly periodic orbit to either the swing-by orbit or the revolving orbit. Because of the order relation for the eigenvalues (32), the stability of the periodic orbits is determined by the second largest eigenvalues $\lambda_{M-1}^{ \pm}$. This is because when $\lambda_{M-1}^{+}$is unstable, the slightly perturbed $N$-ring deviate from the reduced system due to the unstable manifold transverse to the system.

As for the case when the number of the vortex points is odd, linear stability analysis just shows that all the eigenvalues are double and they have linearly independent eigenvectors. It is difficult to derive a simple reduced system as we have done for the even vortex points. It is a future problem to investigate the global dynamics of the unstable $N$-ring consists of odd vortex points.

Finally, we apply the present results to describe the motion of the vortex sheets with rolling-up spirals shown in Figure 1. The linear stability analysis indicates that the vortex sheet with four spirals in Figure 1(a) are unstable, while those with odd spirals in (b), (c), and (d) are neutrally stable. Indeed, according to the paper[16], the vortex sheets with odd spirals rotate stably at the same line of latitude for all the time, while the centers of the four spirals begin changing their relative positions in the latitudinal direction. Moreover, as for the unstable four spirals, since the second largest eigenvalues are neutrally stable, we expect that the spirals evolve like a nonlinearly periodic orbit in theory. However, in reality, since the size of the four rolling-up spirals are different, it is hard to claim it exactly from the simplified $N$-ring model with the same strengths.

\section{Acknowledgements}

This paper is partially supported by Ministry of Education, Science, Sports and Culture, Grand-in-Aid for Young Scientists (B), \#14740069, 2004, and Grand-inAid for formation of COE.

\section{References}

[1] S. Boatto and H.E. Cabral, Nonlinear stability of a latitudinal ring of pointvortices on a nonrotating sphere, SIAM J. Appl. Math., 64(2003), pp. 216-230.

[2] H.E. Cabral and D.S. Schmidt, Stability of relative equilibria in the problem of $N+1$ vortices, SIAM J. Math. Anal., 31(1999), pp. 231-250.

[3] D.G. Dritschel, The stability and energetics of co-rotating uniform vortices, J. Fluid Mech., 358 (1985), pp.95-134.

[4] R. Kidambi and P.K. Newton, Motion of three point vortices on a sphere, Phys. D, 116 (1998), pp.95-134.

[5] R. Kidambi and P.K. Newton, Collapse of three vortices on a sphere, Nouvo Cimento, 22C (1999), pp. 779-791.

[6] R. Kidambi and P.K. Newton, Streamline topologies for integrable vortex motion on a sphere, Physica D, 140 (2000), pp. 95-125.

[7] F. Kirwan, The topology of reduced phase space of the motion of vortices on a sphere, Physica D, 30 (1998), pp. 99-123.

[8] Y. Kimura and H. Okamoto, Vortex motion on a sphere, J. Phys. Soc. Japan, 56 (1987), pp. 4203-4206. 
[9] F. Laurent-Polz, Point vortices on the sphere: A case with opposite vortices, Nonlinearity, 15 (2003), pp. 143-171.

[10] C. Lim, Relative equilibria of symmetric n-body problems on a sphere: inverse and direct results, Comm. Pure. Appl. Math., 51 (1998), pp. 341-371.

[11] C. Lim, J. Montaldi, and M. Roberts, Relative equilibria of point vortices on the sphere, Phys. D, 148 (2001), pp.97-135.

[12] P.K. Newton, The N-vortex problem, Analytical techniques, Springer-Verlag, New York, 2001.

[13] S. Pekarsky and J.E. Marsden, Point vortices on a sphere: Stability of relative equilibria, J. Math. Phys., 39 (1998), pp. 5894-5907.

[14] L.M. Polvani and D.G. Dritschel, Wave and vortex dynamics on the surface of a sphere, J. Fluid Mech., 255 (1993), pp.35-64.

[15] T. Sakajo, The motion of three point vortices on a sphere, Japan J. Indust. Appl. Math., 16 (1999), pp. 321-347.

[16] T. Sakajo, Motion of a vortex sheet on a sphere with pole vortices, Phys. Fluids, 16 (2004), pp. 717-727. 